\title{
Structured Non-uniformly Spaced Rectangular Antenna Array Design for FD-MIMO Systems
}

\author{
Wendong Liu, Zhaocheng Wang, Senior Member, IEEE, Chen Sun, Senior Member, IEEE, \\ Sheng Chen, Fellow, IEEE, and Lajos Hanzo, Fellow, IEEE
}

\begin{abstract}
Full-dimensional multiple-input multiple-output (FD-MIMO) systems, whereby each base station is equipped with a uniformly spaced rectangular antenna array (URA), provides a practical means of realizing massive multiple-input multiple-output systems. However, the spectral efficiency of URA is considerably lower than that of its uniformly spaced linear array counterpart having the same number of antenna elements. In this paper, we first introduce a discrete angular resolution metric for quantifying the low resolution of URA in the antenna-elevation domain. This motivates us to propose a novel antenna device design, referred to as the structured non-uniformly spaced rectangular array (NURA), in which the antenna elements are non-uniformly distributed in the elevationangle domain. Specifically, we conceive a structured NURA device for which the nonuniform distribution of the elevationdomain antenna elements is controlled by a single parameter. The design of the optimally structured NURA for the given nonlinear antenna-element-positioning function then becomes a single-parameter optimization, namely that of maximizing the spectral efficiency of the FD-MIMO system, which can be solved efficiently. Our simulation results demonstrate that our structured NURA design significantly outperforms the standard URA in terms of achievable spectral efficiency. Our proposed structured NURA design therefore offers an effective practical framework for enhancing the achievable performance of FD-MIMO systems.
\end{abstract}

Index Terms-Full-dimension multiple-input multiple-output, Kronecker-product, non-uniformly spaced rectangular antenna array, spectral efficiency, discrete angle resolution, azimuth domain, elevation domain

\section{INTRODUCTION}

Massive multiple-input multiple-output (MIMO) systems is expected to find their way into next-generation wireless communication systems [1]. By equipping the base station (BS) with a large number of antennas, the resultant massive MIMO system benefits from the asymptotic orthogonality of the MIMO channels as the number of antennas at the BS tends to infinity [2]. Therefore, it is capable of dramatically

W. Liu and Z. Wang are with Tsinghua National Laboratory for Information Science and Technology, Department of Electronic Engineering, Tsinghua University, Beijing 100084, China (1wd15@mails.tsinghua.edu.cn, zcwang@tsinghua.edu.cn).

C. Sun is with SONY China Research Laboratory, Beijing 100190, China (chen.sun@sony.com.cn).

S. Chen and L. Hanzo are with Electronics and Computer Science, University of Southampton, Southampton SO17 1BJ, UK (sqc@ecs.soton.ac.uk, lh@ecs.soton.ac.uk). S. Chen is also with King Abdulaziz University,Jeddah 21589, Saudi Arabia.

This work was supported by National Key Basic Research Program of China (No.2013CB329203), National Nature Science Foundation of China (No.61571267), Beijing Natural Science Foundation (No.4142027), National High Technology Research and Development Program of China (No.2014AA01A704), Shenzhen Visible Light Communication System Key Laboratory (ZDSYS20140512114229398) and Shenzhen Peacock Plan (No.1108170036003286) enhancing the achievable spectral efficiency and energy efficiency, whilst only relying on low-complexity linear signal processing methods both for uplink reception and downlink transmission [3], [4]. Although these large-scale linear antenna arrays have an appealing performance potential [5], it is impossible to install such large linear arrays at the BS within a limited space. To circumvent this practical problem, the full-dimensional MIMO (FD-MIMO) concept, also known as the three-dimension MIMO (3D-MIMO) philosophy [6], has become the structure holding the promise of practically constructing massive MIMO systems for the emerging 5th generation $(5 \mathrm{G})$ wireless communication standards. Specifically, FD-MIMOs aim for adapting uniformly spaced rectangular antenna arrays (URA) at the BS. In contrast to the conventional uniformly spaced linear antenna array (ULA), large-scale URA can be readily installed in practice. For example, a $16 \times 16$ halfwavelength spaced URA occupies about $1.2 \mathrm{~m} \times 1.2 \mathrm{~m}$ space when the carrier frequency is $2 \mathrm{GHz}$. By contrast, about $12 \mathrm{~m}$ spacing is required in horizontal direction to install the ULA's 256 antennas. The simulation results of [7] have demonstrated the significant throughput enhancements attained by utilizing massive FD-MIMO systems.

By utilizing URA at the BS, FD-MIMO provides an extra degree of freedom in the elevation-angle domain. Users can now be distinguished not only by their angle-of-arrivals in the azimuth domain (A-AOAs) but also by their angle-of-arrivals in elevation domain (E-AOAs) [5]. Research on establishing appropriate channel models has also been carried out [8], [9], and based on these studies a simplified three-dimension spatial channel model (3D SCM) was introduced in [5]. By performing the Kronecker decomposition of the 3D channel matrix between the BS and a user, the matrix can be expressed as the Kronecker-product (KP) of the azimuth- and elevationangle steering vectors [10], [11]. Moreover, owing to the modest angular spread of the elevation domain in comparison the angular spread of the azimuth domain [8], the multi-path channel model may be approximately simplified to a singlepath one in the elevation domain [11].

Based on the 3D SCM, sophisticated channel estimation and beamforming or precoding algorithms were developed for FDMIMO systems. Note that conventional algorithms based on ULA can also be utilized for URA relying on the vectorization of the channel matrix. However, these conventional methods ignore the structure of the FD-MIMO channel, and their associated complexity increases rapidly as the number of antennas increases. Therefore, vertical elevation-domain beamforming or precoding algorithms have also been discussed in the literature [10], [12]-[16], which exploit the KP property of 3D SCM. In particular, these low-complexity methods rely on 
the elevation-domain-related antennas both for estimating the channels and for distinguishing the users. However, there is a fundamental limitation of URA, namely, the low resolution of the E-AOAs, which leads to the phenomenon that the spectral efficiency gain of URA becomes significantly lower than that of the ULA for the same number of antennas [5]. This limitation of the URA severely restricts the achievable performance of URA based FD-MIMO systems. To resolve this issue, intelligent signal processing algorithms have to be conceived for the FD-MIMO or, alternatively, a completely different nonuniform antenna element topology is necessary.

Against the above background, in this paper, we propose a novel antenna device design, referred to as the structured non-uniformly spaced rectangular array (NURA), in which the antenna elements are non-uniformly distributed in the elevation direction and demonstrate its superior spectral efficiency compared to the standard URA design. Our main contributions are summarized below.

- We propose a generic discrete angular resolution (DAR) metric, which quantifies the antenna array's angular resolutions in both the azimuth- and elevation-directions and show that the resolution of the URA for E-AOAs can be an order of magnitude lower than that for A-AOAs. This reveals the root cause of the URA's lower performance compared to the ULA having the same number of antenna elements and motivates the search for alternative antenna arrays relying on nonuniform devices in the elevation domain.

- To make the problem sufficiently simple and tractable while retaining the beneficial KP-structure of the $3 \mathrm{D}$ SCM, we specifically consider the structured NURA device for which the nonuniform distribution of elevationdomain antenna elements is controlled by a single parameter. The standard URA becomes a specific case of this structured NURA associated with this control parameter assuming a specific value.

- The design of the optimally structured NURA for the given nonlinear antenna-element-positioning function is then turned into a single-parameter optimization problem, which maximizes the spectral efficiency of the FD-MIMO system based on the specifically designed structured NURA device. We demonstrate that this problem can be efficiently solved. Our simulation results demonstrate that our structured NURA significantly outperforms the standard URA in terms of achievable spectral efficiency.

The structured NURA design proposed therefore provides an effective framework for enhancing the achievable performance of FD-MIMO.

\section{Three-Dimension Spatial Channel Model}

We first briefly introduce the 3D SCM, which is universally utilized in FD-MIMO systems, followed by discussing the KPstructure of the channel matrix generated by URA. In order to interpret the lower performance of URA in comparison to a ULA associated with the same total number of antenna elements, the low resolution of the URA for E-AOAs is demonstrated in the discrete angular domain by means of the

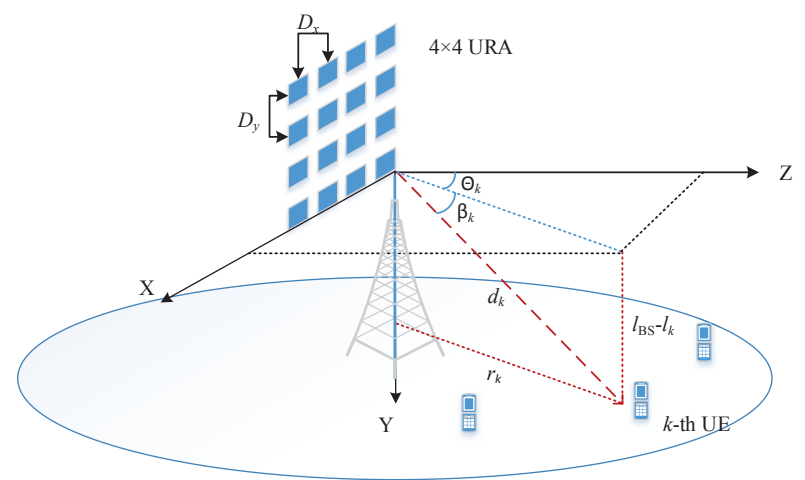

Fig. 1. Illustration of the single-cell multi-user FD-MIMO system with a URA.

discrete Fourier transform (DFT). The issues of downlink data transmission are then summarized for the FD-MIMO system. For notational simplification, we use the multi-user single-cell senario to illustrate the underlying principles.

\section{A. Channel Matrix and Kronecker-product Structure}

As illustrated in Fig. 1, a population of $K$ singleantenna user equipments (UEs) are served with the same time/frequency resource by the BS, which is equipped with a URA having $M_{y}$ and $M_{x}$ antenna elements in the elevationand azimuth-domains, respectively. In Fig. $1, l_{\mathrm{BS}}$ is the BS's height, $l_{k}$ is the $k$-th UE's height, $r_{k}$ and $d_{k}$ denote the horizontal distance and distance between the BS and the $k$-th user, respectively, while $\theta_{k}$ and $\beta_{k}$ are the the A-AOA and E-AOA of the $k$-th UE, respectively. Additional, we use $D_{x}$ and $D_{y}$ to denote the antenna spacings in the azimuth- and elevation-domains, respectively.

The channel matrix between the BS and the $k$-th user is denoted by $\mathbf{H}_{k} \in \mathbb{C}^{M_{y} \times M_{x}}$. We assume to encounter a non-dispersive narrow-band multi-path channel, where $\mathbf{H}_{k}$ is expressed as

$$
\mathbf{H}_{k}=\sum_{p=1}^{P} \mathbf{H}_{k}^{p},
$$

in which $P$ is the number of paths and $\mathbf{H}_{k}^{p}$ is the $p$-th path's channel matrix. Further assume $D_{x}=D_{y}=D$ for simplicity. The element at the $l$-th row and $m$-th column of $\mathbf{H}_{k}^{p}$ is given by [5]

$$
\begin{aligned}
h_{k}^{p, m, l}= & \rho_{k}^{p} \frac{1}{\sqrt{M_{x} M_{y}}} e^{-\mathrm{j} 2 \pi \frac{D}{\lambda}\left((m-1) \cos \theta_{k}^{p} \cos \beta_{k}^{p}+(l-1) \sin \beta_{k}^{p}\right)} \\
= & \rho_{k}^{p}\left(\frac{1}{\sqrt{M_{x}}} e^{-\mathrm{j} 2 \pi \frac{(m-1) D}{\lambda} \cos \theta_{k}^{p} \cos \beta_{k}^{p}}\right) \\
& \times\left(\frac{1}{\sqrt{M_{y}}} e^{-\mathrm{j} 2 \pi \frac{(l-1) D}{\lambda} \sin \beta_{k}^{p}}\right)=\rho_{k}^{p} h_{\mathrm{a}, k}^{p, m} h_{\mathrm{e}, k}^{p, l},
\end{aligned}
$$

where $\mathrm{j}=\sqrt{-1}, \lambda$ is the wavelength, $\theta_{k}^{p}$ and $\beta_{k}^{p}$ are the A-AOA and E-AOA of the $p$-th path, respectively, while $\rho_{k}^{p}$ represents the path's large-scale fading coefficient, which is calculated according to

$$
\rho_{k}^{p}=\frac{z_{k}^{p}}{\left(d_{k}\right)^{\gamma}} .
$$


In the formula (3), $\gamma$ is the path-loss exponent and $z_{k}^{p}$ is the shadow-fading coefficient, which follows the log-normal distribution $\ln z_{k}^{p} \sim \mathcal{N}\left(0, \sigma_{z}^{2}\right)$ with variance $\sigma_{z}^{2}$.

Observe from (2) that each element in $\mathbf{H}_{k}^{p}$ can be expressed as the product of two terms, $h_{\mathrm{a}, k}^{p, m}$ and $h_{\mathrm{e}, k}^{p, l}$, which can be regarded as the azimuth and elevation components of $h_{k}^{p, m, l}$, respectively. Using this decomposition, the channel matrix $\mathbf{H}_{k}^{p}$ is expressed as

$$
\mathbf{H}_{k}^{p}=\rho_{k}^{p} \mathbf{h}_{\mathrm{e}, k}^{p} \otimes\left(\mathbf{h}_{\mathrm{a}, k}^{p}\right)^{\mathrm{T}},
$$

where $\otimes$ represents the KP operator and $(\cdot)^{\mathrm{T}}$ is the transpose operator, while

$$
\begin{aligned}
\mathbf{h}_{\mathrm{e}, k}^{p} & =\frac{1}{\sqrt{M_{y}}}\left[1 h_{\mathrm{e}, k}^{p, 1} \cdots h_{\mathrm{e}, k}^{p, M_{y}}\right]^{\mathrm{T}} \in \mathbb{C}^{M_{y} \times 1}, \\
\mathbf{h}_{\mathrm{a}, k}^{p} & =\frac{1}{\sqrt{M_{x}}}\left[\begin{array}{ll}
1 & h_{\mathrm{a}, k}^{p, 1} \cdots h_{\mathrm{a}, k}^{p, M_{x}}
\end{array}\right]^{\mathrm{T}} \in \mathbb{C}^{M_{x} \times 1},
\end{aligned}
$$

are the elevation- and azimuth-domain steering vectors, respectively. Then the overall channel matrix can be rewritten as

$$
\mathbf{H}_{k}=\sum_{p=1}^{P} \rho_{k}^{p} \mathbf{h}_{\mathrm{e}, k}^{p} \otimes\left(\mathbf{h}_{\mathrm{a}, k}^{p}\right)^{\mathrm{T}} .
$$

Utilizing the KP-based 3D SCM, an eigenvalue beamforming scheme was proposed in [10] for the elevation-domain, while KP-based codebooks and a reference signal (RS) was conceived for a FD-MIMO aided LTE-A system [10], [14][16], under the assumption that the angular spread experienced in the elevation domain is small so that the elevation-domain steering vectors of multi-path scenarios can be approximated by a single-path vector [8]. With this approximation, the KP structure of $\mathbf{H}_{k}$ is represented by two separate parts as follows

$$
\mathbf{H}_{k} \approx \mathbf{h}_{\mathrm{e}, k} \otimes \sum_{p=1}^{P} \rho_{k}^{p} \mathbf{h}_{\mathrm{a}, k}^{p},
$$

where $\mathbf{h}_{\mathrm{e}, k} \approx \mathbf{h}_{\mathrm{e}, k}^{p}$ for $1 \leq p \leq P$ is the approximate single-path steering vector in the elevation-domain. Many existing techniques rely on this approximate KP structure to perform channel estimation and beamforming/precoding in the elevation and azimuth domains separately, which enjoys a lowcomplexity compared to conventional methods operating on the vectorization of $\mathbf{H}_{k}$ without exploiting this approximate KP structure. However, in some scenarios, the angular spread experienced in the elevation-domain may be high and the performance of these low-complexity schemes would degrade, because the approximate KP structure of (8) may become inaccurate. It should be emphasized that the approximate KP structure (8) is only presented to introduce the low-complexity KP-based elevation beamforming/precoding algorithms. The design of our NURA and the channel generation in the simulation study are based on the full channel model (7).

\section{B. Discrete Angle Resolution}

If the signals arriving at the BS from $K$ users have sufficiently different E-AOAs and/or A-AOAs, the BS can readily rely on beamforming/precoding for efficiently reducing the inter-user interference (IUI). However, if the users' E-AOAs and A-AOAs are similar in the spatial domain or, worse still, they overlap, then their discrete angular spectrum cannot be separated in the discrete angular domain by DFT, which leads to inaccuracy in channel estimation and degrades the efficiency of the beamforming/precoding operation. For characterizing the degree of separation in the discrete angular domain, we define the average DAR in the discrete angular domain.

1) Average discrete azimuth angle resolution: First, let us consider a multi-user MIMO employing an $M$-element ULA in the horizontal direction at the BS. In this case, only the A-AOAs of the users are relevant. According to [17], the channel steering vector at the A-AOA $\theta, \mathbf{a}(\theta)=$ $\left[1 e^{-\mathrm{j} 2 \pi \frac{D}{\lambda} \cos \theta} \cdots e^{-\mathrm{j} 2 \pi \frac{(M-1) D}{\lambda} \cos \theta}\right]^{\mathrm{T}} \in \mathbb{C}^{M \times 1}$, can be treated as a single-frequency signal with the frequency $f_{\mathrm{a}}=\frac{D}{\lambda} \cos \theta$. The $N$-points DFT of $\mathbf{a}(\theta)$ is calculated according to

$$
\mathbf{b}=\mathbf{F a}(\theta),
$$

where $N \geq M, \mathbf{F} \in \mathbb{C}^{N \times M}$ is the DFT matrix and $\mathbf{b} \in \mathbb{C}^{N \times 1}$ is the angle spectrum vector in discrete angle domain. Denote $n_{\max }$ as the index of the element of $\mathbf{b}$ whose magnitude attains $\|\mathbf{b}\|_{l_{\infty}}$, i.e., $\left|b_{n_{\max }}\right|=\|\mathbf{b}\|_{l_{\infty}}$, where $\|\cdot\|_{l_{\infty}}$ represents the $l_{\infty^{-}}$ norm operator. From [17], we have

$$
\left|b_{n_{\max }}\right|=\left|\sum_{m=0}^{M-1} e^{-\mathrm{j} 2 \pi m Q\left(n_{\max }\right)}\right| \simeq M,
$$

and

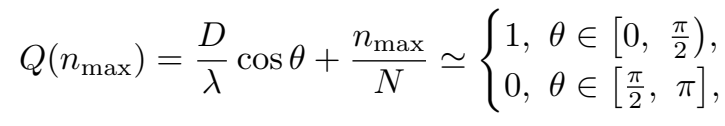

where the notation ' $\simeq$ ' denotes equal to ' $=$ ' or approximately equal to ' $\approx$ '. With this definition of $\simeq$, the interpretation of (10) and (11) is obvious [17]. Only when $Q\left(n_{\max }\right) \in \mathbb{Z}$, we can obtain the maximum value of $\left|b_{n_{\max }}\right|=M$. For any other value of $Q\left(n_{\max }\right)$, we only have the value of $\left|b_{n_{\max }}\right|$ close to $M$, i.e., $\left|b_{n_{\max }}\right| \approx M$. Generally, the A-AOA $\theta$ is uniformly distributed in $[0, \pi]$. Considering that the element spacing of the ULA is half the wavelength, i.e., $\frac{D}{\lambda}=\frac{1}{2}$, we have $Q(n) \in\left(0, \frac{3}{2}\right]$ for $\theta \in\left[0, \frac{\pi}{2}\right)$ and $Q(n) \in\left[-\frac{1}{2}, \frac{1}{2}\right]$ for $\theta \in\left[\frac{\pi}{2}, \pi\right]$. Thus, for $\theta \in\left[0, \frac{\pi}{2}\right)$, we have $Q\left(n_{\max }\right) \simeq 1$, while for $\theta \in\left[\frac{\pi}{2}, \pi\right]$, we have $Q\left(n_{\max }\right) \simeq 0$. From (11), we further have

$$
n_{\max } \simeq \begin{cases}N\left(1-\frac{D}{\lambda} \cos \theta\right), & \theta \in\left[0, \frac{\pi}{2}\right), \\ -N \frac{D}{\lambda} \cos \theta, & \theta \in\left[\frac{\pi}{2}, \pi\right] .\end{cases}
$$

It can be seen from (12) that signals having different A-AOAs $\theta$ achieve their peak values at different positions $n_{\max }$. Thus, for two users with A-AOAs $\theta_{1}$ and $\theta_{2}$ attaining their peak values at $n_{\text {max }_{1}}$ and $n_{\max _{2}}$, respectively, we may quantify the separation of the two users in the discrete angular domain by $\left|n_{\max _{1}}-n_{\max _{2}}\right|$. Note that $n_{\max }$ is discontinuous at $\theta=\frac{\pi}{2}$ and naturally, if two users are just located at the two sides of $\frac{\pi}{2},\left|n_{\max _{1}}-n_{\max _{2}}\right|$ should be very small. To take into account this circularity of DFT, we define

$$
n_{\max }^{\prime}= \begin{cases}n_{\max }, & \theta \in\left[0, \frac{\pi}{2}\right], \\ n_{\max }+N, & \theta \in\left[\frac{\pi}{2}, \pi\right],\end{cases}
$$


and revise the indexes for the peak values of the two signals according to $n_{\max _{1}}^{\prime}$ and $n_{\max _{2}}^{\prime}$, respectively. Then $\Delta\left(\theta_{1}, \theta_{2}\right)=$ $\left|n_{\max _{1}}^{\prime}-n_{\max _{2}}^{\prime}\right|$ is a proper measure of the DAR in the discrete angular domain for the two users. A larger $\Delta\left(\theta_{1}, \theta_{2}\right)$ indicates that the two users can be separated more clearly. Since the users are randomly distributed and exhibit a multi-path angular spread, the expectation of $\Delta$ is used to measure the average DAR in the azimuth-domain (A-DAR), which was formerly defined as

$$
\begin{aligned}
\bar{\Delta}^{\mathrm{a}} & =E\left\{\left|n_{\max _{1}}^{\prime}-n_{\max _{2}}^{\prime}\right|\right\} \\
& \simeq \int_{0}^{\pi} \int_{0}^{\pi} \Delta\left(\theta_{1}, \theta_{2}\right) p\left(\theta_{1}, \theta_{2}\right) \mathrm{d} \theta_{1} \mathrm{~d} \theta_{2},
\end{aligned}
$$

where $E\{\cdot\}$ denotes the expectation operator and $p\left(\theta_{1}, \theta_{2}\right)$ is the joint probability density function (PDF) of $\left(\theta_{1}, \theta_{2}\right)$. Under the realistic assumption that $\theta_{1}$ and $\theta_{2}$ are independent and both follow the uniform distribution $\mathcal{U}[0, \pi], p\left(\theta_{1}, \theta_{2}\right)=$ $p\left(\theta_{1}\right) p\left(\theta_{2}\right)=\frac{1}{\pi^{2}}$. Also noting (13), we have

$$
\begin{aligned}
\bar{\Delta}^{\mathrm{a}} \simeq & \frac{N D}{\lambda \pi^{2}} \int_{0}^{\pi} \int_{0}^{\pi}\left|\cos \theta_{1}-\cos \theta_{2}\right| \mathrm{d} \theta_{1} \mathrm{~d} \theta_{2} \\
= & \frac{N D}{\lambda \pi^{2}}\left(\operatorname{Pr}\left(\theta_{1}<\theta_{2}\right) \int_{0}^{\pi} \int_{0}^{\theta_{2}}\left(\cos \theta_{1}-\cos \theta_{2}\right) \mathrm{d} \theta_{1} \mathrm{~d} \theta_{2}\right. \\
& \left.+\operatorname{Pr}\left(\theta_{2}<\theta_{1}\right) \int_{0}^{\pi} \int_{0}^{\theta_{1}}\left(\cos \theta_{2}-\cos \theta_{1}\right) \mathrm{d} \theta_{2} \mathrm{~d} \theta_{1}\right)
\end{aligned}
$$

By exploiting the symmetry of $\theta_{1}$ and $\theta_{2}, \operatorname{Pr}\left(\theta_{1}<\theta_{2}\right)=$ $\operatorname{Pr}\left(\theta_{2}<\theta_{1}\right)=\frac{1}{2}$, we have

$$
\bar{\Delta}^{\mathrm{a}} \simeq \frac{N D}{\lambda \pi^{2}} \int_{0}^{\pi} \int_{0}^{\theta_{2}}\left(\cos \theta_{1}-\cos \theta_{2}\right) \mathrm{d} \theta_{1} \mathrm{~d} \theta_{2}=\frac{4 N D}{\lambda \pi^{2}} .
$$

For half-wavelength array element spacing, we further have $\bar{\Delta}^{\mathrm{a}} \simeq \frac{2}{\pi^{2}} N \approx 0.2 N$.

2) Average discrete elevation angle resolution: Next consider the same ULA, but invoked for the vertical direction. In this case, only the E-AOAs of users are relevant. The channel's steering vector at the E-AOA $\beta, \mathbf{e}(\beta)=$ $\left[1 e^{-\mathrm{j} 2 \pi \frac{D}{\lambda} \sin \beta} \cdots e^{-\mathrm{j} 2 \pi \frac{(M-1) D}{\lambda} \sin \beta}\right]^{\mathrm{T}} \in \mathbb{C}^{M \times 1}$ can also be seen as a single-frequency signal with the frequency $f_{\mathrm{e}}=$ $\frac{D}{\lambda} \sin \beta$. Define $\mathbf{c}=\mathbf{F e}(\beta)$, and let $n_{\max }$ be the index of the element of $\mathbf{c}$ whose magnitude attains $\|\mathbf{c}\|_{l_{\infty}}$. Similarly, we have

$$
\left|c_{n_{\max }}\right|=\left|\sum_{m=0}^{M-1} e^{-\mathrm{j} 2 \pi m P\left(n_{\max }\right)}\right| \simeq M
$$

with

$$
P\left(n_{\max }\right)=\frac{D}{\lambda} \sin \beta+\frac{n_{\max }}{N} \simeq 1 .
$$

The maximum value $M$ is attained only when $P\left(n_{\max }\right) \in \mathbb{Z}$, while for other values of $P\left(n_{\max }\right)$, we only have $\left|c_{n_{\max }}\right| \approx$ $M$. Since the user height $l$ is much lower than $l_{\mathrm{BS}}$, the E-AOA obeys $\beta \in\left[\beta_{\min }, \beta_{\max }\right] \subset\left(0, \frac{\pi}{2}\right)$. That is, the minimum EAOA $\beta_{\min }>0$ and the maximum E-AOA $\beta_{\max }<\frac{\pi}{2}$. As a result, $P(n) \in\left(0, \frac{3}{2}\right)$. Hence, in contrast to $(11), P\left(n_{\max }\right)$ is always equal or close to 1 , i.e., $P\left(n_{\max }\right) \simeq 1$. Furthermore, we have $n_{\max } \simeq N\left(1-\frac{D}{\lambda} \sin \beta\right)$.
In general, $l$ is a random variable obeying some unknown distribution. For analytical tractability, let us assume that the difference $l^{\prime}=l_{\mathrm{BS}}-l$ between the $\mathrm{BS}$ height $l_{\mathrm{BS}}$ and user height $l$ is constant. Then it is straightforward to show that the $\mathrm{PDF}$ of $\beta$ is given by

$$
p(\beta)=\left\{\begin{array}{cl}
\frac{l^{\prime} \csc ^{2} \beta}{r_{\max }-r_{\min }}, & \beta_{\min } \leq \beta \leq \beta_{\max } \\
0 & \text { otherwise }
\end{array}\right.
$$

where $r_{\max }=\frac{l^{\prime}}{\tan \beta_{\min }}$ and $r_{\min }=\frac{l^{\prime}}{\tan \beta_{\max }}$. Let $\beta_{1}$ and $\beta_{2}$ be the E-AOAs from two users. Similarly, we can define their elevation-domain DAR (E-DAR) $\Delta\left(\beta_{1}, \beta_{2}\right)$ and compute the average E-DAR according to

$$
\bar{\Delta}^{\mathrm{e}} \simeq \int_{\beta_{\min }}^{\beta_{\max }} \int_{\beta_{\min }}^{\beta_{\max }} \Delta\left(\beta_{1}, \beta_{2}\right) p\left(\beta_{1}, \beta_{2}\right) \mathrm{d} \beta_{1} \mathrm{~d} \beta_{2},
$$

where $p\left(\beta_{1}, \beta_{2}\right)$ is the joint PDF of $\left(\beta_{1}, \beta_{2}\right)$. Since $\beta_{1}$ and $\beta_{2}$ are independent, $p\left(\beta_{1}, \beta_{2}\right)=p\left(\beta_{1}\right) p\left(\beta_{2}\right)$. Thus, similar to the calculation of $\Delta^{\mathrm{a}}, \Delta^{\mathrm{e}}$ can be simplified as

$$
\begin{aligned}
\bar{\Delta}^{\mathrm{e}} \simeq & \frac{N D\left(l^{\prime}\right)^{2}}{\lambda\left(r_{\max }-r_{\min }\right)^{2}} \times \\
& \int_{\beta_{\min }}^{\beta_{\max }} \int_{\beta_{\min }}^{\beta_{1}}\left(\sin \beta_{1}-\sin \beta_{2}\right) \csc ^{2} \beta_{2} \csc ^{2} \beta_{1} \mathrm{~d} \beta_{2} \mathrm{~d} \beta_{1} \\
= & \frac{N D\left(l^{\prime}\right)^{2}}{\lambda\left(r_{\max }-r_{\min }\right)^{2}}\left(\left(\cot \beta_{\min }+\cot \beta_{\max }\right)\right. \\
& \times \ln \left|\frac{\csc \beta_{\max }-\cot \beta_{\max }}{\csc \beta_{\min }-\cot \beta_{\min }}\right| \\
& \left.+2\left(\csc \beta_{\max }-\csc \beta_{\min }\right)\right) .
\end{aligned}
$$

In particular, for half-wavelength array element spacing, we have $\frac{D}{\lambda}=0.5$.

3) Average Azimuth- and Elevation-Domain Discrete Angular Resolution of URA: For FD-MIMO systems adopting the URA with $M_{y}=M$ elevation antenna elements and $M_{x}=M$ azimuth antenna elements, the azimuth and elevation steering vectors of a user associated with A-AOA $\theta$ and E-AOA $\beta$ are

$$
\begin{aligned}
\mathbf{a}(\theta, \beta) & =\left[1 e^{-\mathrm{j} 2 \pi \frac{D}{\lambda} \cos \theta \cos \beta} \cdots e^{-\mathrm{j} 2 \pi \frac{(M-1) D}{\lambda} \cos \theta \cos \beta}\right]^{\mathrm{T}} \\
& \approx\left[1 e^{-\mathrm{j} 2 \pi \frac{D}{\lambda} \cos \theta} \cdots e^{-\mathrm{j} 2 \pi \frac{(M-1) D}{\lambda} \cos \theta}\right]^{\mathrm{T}} \\
& =\mathbf{a}(\theta) \\
\mathbf{e}(\beta) & =\left[1 e^{-\mathrm{j} 2 \pi \frac{D}{\lambda} \sin \beta} \cdots e^{-\mathrm{j} 2 \pi \frac{(M-1) D}{\lambda} \sin \beta}\right]^{\mathrm{T}}
\end{aligned}
$$

respectively. The approximation in (22) corresponds to ignoring the coupling effects between the elevation and azimuth domains, which is reasonable considering that in the generic azimuth component $e^{-\mathrm{j} 2 \pi \frac{(m-1) D}{\lambda} \cos \theta \cos \beta}, \beta$ is much smaller than $\theta$ and consequently $\cos \beta$ is close to 1 . Clearly, we can use $\bar{\Delta}^{\mathrm{e}}$ of (21) to measure the average E-DAR of the URA, and we may approximate the average A-DAR of the URA by $\bar{\Delta}^{\mathrm{a}}$ of (16).

Consider a practical FD-MIMO system, where we have $l^{\prime}=$ $35 \mathrm{~m}, r_{\min }=50 \mathrm{~m}$ and $r_{\max }=200 \mathrm{~m}$. Thus $\beta_{\min } \approx 0.173$ and $\beta_{\max } \approx 0.611$. With half-wavelength array element spacing, we have $\bar{\Delta}^{\mathrm{e}} \approx 0.02 N$ but $\bar{\Delta}^{\mathrm{a}} \approx 0.2 N=10 \bar{\Delta}^{\mathrm{e}}$. Thus in this case the A-DAR is an order of magnitude more accurate than the E-DAR, which indicates that the performance of the URA 
in the azimuth domain is much better than that in the elevation domain. This result explains why the performance of the FDMIMO system equipped with the URA suffers from severe performance loss compared to the convention MIMO adopting the ULA of the same total number of antenna elements, as observed in the simulation results of [5]. If the height of the BS is increased, the E-DAR will be increased. However, the height of the BS is typically constrained. Therefore, to fundamentally solve the problem of low E-DAR, carefully constructed new antenna arrays should be designed in order to mitigate the performance loss of FD-MIMO systems caused by low EDAR, which motivates our current study.

\section{Downlink Data Transmission}

Before we investigate new array designs, let us summarize the downlink data transmission in the FD-MIMO system. For the channel matrix between the BS and the $k$-th user, $\mathbf{H}_{k}$, let us define $\overline{\mathbf{h}}_{k}=\operatorname{vec}\left(\mathbf{H}_{k}\right) \in \mathbb{C}^{\left(M_{y} M_{x}\right) \times 1}$, where $\operatorname{vec}(\cdot)$ denotes the vector stacking operator. The whole channel matrix between the BS and the $K$ users can then be expressed as $\mathbf{H}=\left[\overline{\mathbf{h}}_{1} \overline{\mathbf{h}}_{2} \cdots \overline{\mathbf{h}}_{K}\right]^{\mathrm{T}} \in \mathbb{C}^{K \times\left(M_{y} M_{x}\right)}$. Denote $\mathbf{x}=\left[\begin{array}{lll}x_{1} & x_{2} \cdots x_{K}\end{array}\right]^{\mathrm{T}} \in \mathbb{C}^{K \times 1}$ with $E\left\{\mathbf{x x}^{\mathrm{H}}\right\}=\mathbf{I}_{K}$ as the symbol vector transmitted by the BS to the $K$ users, where $(\cdot)^{\mathrm{H}}$ denotes the conjugate transpose operator and $\mathbf{I}_{K}$ is the $K \times K$ identity matrix. The data received by the $K$ users,

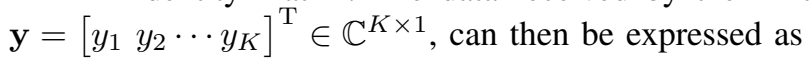

$$
\mathbf{y}=\mathbf{H W} \mathbf{x}+\mathbf{n},
$$

where $\mathbf{n} \in \mathbb{C}^{K \times 1}$ is the complex additive white Gaussian noise (AWGN) vector with $E\left\{\mathbf{n n}^{\mathrm{H}}\right\}=\sigma_{n}^{2} \mathbf{I}_{K}$, and $\mathbf{W}=$ $\left[\mathbf{w}_{1} \mathbf{w}_{2} \cdots \mathbf{w}_{K}\right] \in \mathbb{C}^{\left(M_{y} M_{x}\right) \times K}$ is the precoding matrix, in which $\mathbf{w}_{k} \in \mathbb{C}^{\left(M_{y} M_{x}\right) \times 1}$ is the precoding vector for the $k$-th user. Hence, for the $k$-th user, its received symbol is given by

$$
y_{k}=\overline{\mathbf{h}}_{k}^{\mathrm{T}} \mathbf{w}_{k} x_{k}+\overline{\mathbf{h}}_{k}^{\mathrm{T}} \sum_{k^{\prime} \neq k} \mathbf{w}_{k^{\prime}} x_{k^{\prime}}+n_{k},
$$

where the first term is the desired signal, the second term is the IUI, and the third term is the noise element. Since the FDMIMO is interference limited [3], we will ignore the influence of $n_{k}$ and concentrate on the desired signal-to-interference ratio (SIR). The SIR of the $k$-th user is given by

$$
\operatorname{SIR}_{k}=\frac{\left|\overline{\mathbf{h}}_{k}^{\mathrm{T}} \mathbf{w}_{k}\right|^{2}}{\sum_{k^{\prime} \neq k}\left|\overline{\mathbf{h}}_{k}^{\mathrm{T}} \mathbf{w}_{k^{\prime}}\right|^{2}} .
$$

The spectral efficiency of the $k$-th user associated with $\mathrm{SIR}_{k}$ is defined as

$$
C_{k}=\log _{2}\left(1+\mathrm{SIR}_{k}\right) .
$$

Numerous precoding algorithms have been proposed for optimizing the average spectral efficiency $C=\frac{1}{K} \sum_{k=1}^{K} C_{k}$. For the ideal zero-forcing $(\mathrm{ZF})$ precoding based on perfect channel estimates, $\mathbf{h}_{k}^{\mathrm{T}} \mathbf{w}_{k^{\prime}}=0$ for $k \neq k^{\prime}$, and the IUI is completely removed. The $\mathrm{ZF}$ precoding weight vector is calculated according to [10], [14]-[16]

$$
\mathbf{w}_{k}^{\mathrm{ZF}}=\mathbf{v e c}\left(\mathbf{w}_{\mathrm{a}, k}^{\mathrm{ZF}} \otimes \mathbf{w}_{\mathrm{e}, k}^{\mathrm{ZF}}\right),
$$

where

$$
\begin{aligned}
\mathbf{w}_{\mathrm{e}, k}^{\mathrm{ZF}} & =\gamma_{\mathrm{e}, k}^{\mathrm{ZF}}\left\langle\mathbf{H}_{\mathrm{e}}^{\mathrm{H}}\left(\mathbf{H}_{\mathrm{e}} \mathbf{H}_{\mathrm{e}}^{\mathrm{H}}\right)^{-1}\right\rangle_{k}, \\
\mathbf{w}_{\mathrm{a}, k}^{\mathrm{ZF}} & =\gamma_{\mathrm{a}, k}^{\mathrm{ZF}}\left\langle\mathbf{H}_{\mathrm{a}}^{\mathrm{H}}\left(\mathbf{H}_{\mathrm{a}} \mathbf{H}_{\mathrm{a}}^{\mathrm{H}}\right)^{-1}\right\rangle_{k},
\end{aligned}
$$

in which $\gamma_{\mathrm{e}, k}^{\mathrm{ZF}}=\left\|\left\langle\mathbf{H}_{\mathrm{e}}^{\mathrm{H}}\left(\mathbf{H}_{\mathrm{e}} \mathbf{H}_{\mathrm{e}}^{\mathrm{H}}\right)^{-1}\right\rangle_{k}\right\|_{2}^{-1}$ and $\gamma_{\mathrm{a}, k}^{\mathrm{ZF}}=$ $\left\|\left\langle\mathbf{H}_{\mathrm{a}}^{\mathrm{H}}\left(\mathbf{H}_{\mathrm{a}} \mathbf{H}_{\mathrm{a}}^{\mathrm{H}}\right)^{-1}\right\rangle_{k}\right\|_{2}^{-1}$ are the power normalizing factors for the elevation- and azimuth-domain precoders, respectively, to ensure the equal power allocation, and $\langle\mathbf{X}\rangle_{k}$ denotes the $k$-th column of $\mathbf{X}$, while

$$
\begin{aligned}
& \mathbf{H}_{\mathrm{e}}=\left[\begin{array}{lll}
\mathbf{h}_{\mathrm{e}, 1} & \mathbf{h}_{\mathrm{e}, 2} \cdots \mathbf{h}_{\mathrm{e}, K}
\end{array}\right]^{\mathrm{T}} \in \mathbb{C}^{K \times M_{y},} \\
& \mathbf{H}_{\mathrm{a}}=\left[\begin{array}{lll}
\mathbf{h}_{\mathrm{a}, 1} & \mathbf{h}_{\mathrm{a}, 2} \cdots \mathbf{h}_{\mathrm{a}, K}
\end{array}\right]^{\mathrm{T}} \in \mathbb{C}^{K \times M_{x},}
\end{aligned}
$$

with

$$
\mathbf{h}_{\mathrm{a}, k}=\mathbf{H}_{k}^{\mathrm{T}} \mathbf{w}_{\mathrm{e}, k}^{\mathrm{ZF}}, 1 \leq k \leq K .
$$

Using a beamforming/precoding scheme based on the approximate KP-structure of the 3D SCM, however, the IUI cannot be completely eliminated. For massive FD-MIMOs, the asymptotic orthogonality will ensure that even employing low-complexity matched filter (MF) based precoding, we can reduce the IUI to a sufficiently low level [18], [19]. The MF precoding weight vector is given by [10], [14]-[16]

$$
\mathbf{w}_{k}^{\mathrm{MF}}=\operatorname{vec}\left(\mathbf{w}_{\mathrm{a}, k}^{\mathrm{ZF}} \otimes \mathbf{w}_{\mathrm{e}, k}^{\mathrm{MF}}\right)
$$

with

$$
\mathbf{w}_{\mathrm{e}, k}^{\mathrm{MF}}=\gamma_{\mathrm{e}, k}^{\mathrm{MF}} \mathbf{h}_{\mathrm{e}, k}^{\mathrm{H}},
$$

where $\gamma_{\mathrm{e}, k}^{\mathrm{MF}}=\left\|\mathbf{h}_{\mathrm{e}, k}^{\mathrm{H}}\right\|_{2}^{-1}$ is the corresponding power normalizing factor for the elevation precoder.

\section{Proposed Structured NURA Design}

As argued in the previous section, the low E-DAR of the URA is the underlying cause that limits the achievable performance of the URA based FD-MIMO. We propose an alternative NURA device in which the antenna elements are non-uniformly allocated in the elevation domain. Based on the aforementioned 3D SCM and on the downlink transmission model, our objective is to design the configuration of our NURA to maximize the average spectral efficiency.

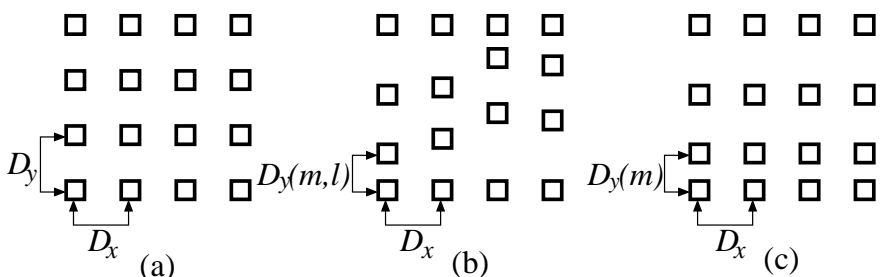

Fig. 2. (a) $4 \times 4$ URA, (b) $4 \times 4$ unstructured NURA in elevation domain, and (c) $4 \times 4$ structured NURA in elevation domain.

\section{A. Structured NURA}

In the URA, the spacings between adjacent antenna elements in azimuth and elevation directions are both constant, denoted by $D_{x}$ and $D_{y}$, respectively, as illustrated in Fig. 2 (a). Usually we set $D_{x}=D_{y}=D$. There are two generic NURA designs with the antenna elements non-uniformly spaced in the elevation domain, as shown in Fig. 2 (b) and (c). For the 
unstructured NURA of Fig. 2(b), the elevation-domain array element spacing depends on both the row index and column index of the array. By contrast, in the structured NURA of Fig. 2 (c), the elevation array element spacing depends only on the row of the array, and every elevation subarray has an identical nonuniform distribution. To make the problem sufficiently tractable and to maintain the KP-structure of the 3D SCM, we only consider the structured NURA.

1) Generic nonlinear element-positioning function: Denote the indices of the antenna elements by $m=0,1, \cdots, M_{y}-1$ in the elevation-domain and the positions of the elevationdomain antenna elements by $f(m)$ for $0 \leq m \leq M_{y}-1$, respectively. Then with the generic nonlinear element-position function $f(m)$ defined by

$$
\begin{aligned}
0=f(0) & <f(1)<\cdots<f\left(M_{y}-2\right) \\
& <f\left(M_{y}-1\right)=D\left(M_{y}-1\right),
\end{aligned}
$$

we can specify the generic NURA structure, whose elevationdomain antenna elements are non-uniformly distributed but still holds the same overall spatial size. By choosing different nonlinear element-position functions, we arrive at differently structured NURAs. Upon opting for a linear elementpositioning function $f(m)=m D$ for $0 \leq m \leq M_{y}-1$, we arrive at the URA.

For the structured NURA relying on a nonlinear elementpositioning function (36), the corresponding elevation-domain steering vector of user $k, \widetilde{\mathbf{h}}_{\mathrm{e}, k}^{p} \in \mathbb{C}^{M_{y} \times 1}$, can be rewritten as

$$
\begin{gathered}
\widetilde{\mathbf{h}}_{\mathrm{e}, k}^{p}=\frac{1}{\sqrt{M_{y}}}\left[1 e^{-\mathrm{j} 2 \pi \frac{f(1)}{\lambda} \sin \beta_{k}^{p}} \cdots e^{-\mathrm{j} 2 \pi \frac{f(m)}{\lambda} \sin \beta_{k}^{p}}\right. \\
\left.\cdots e^{-\mathrm{j} 2 \pi \frac{f\left(M_{y}-1\right)}{\lambda} \sin \beta_{k}^{p}}\right]^{\mathrm{T}} .
\end{gathered}
$$

Combined with the same azimuth-domain steering vector $\mathbf{h}_{\mathrm{a}, k}^{p}$, the channel matrix $\widetilde{\mathbf{H}}_{k} \in \mathbb{C}^{M_{y} \times M_{x}}$ of user $k$ is given by a similar KP operation:

$$
\widetilde{\mathbf{H}}_{k}=\sum_{p=1}^{P} \rho_{k}^{p} \widetilde{\mathbf{h}}_{\mathrm{e}, k}^{p} \otimes \mathbf{h}_{a, k}^{p} .
$$

Let $\widetilde{\mathbf{e}}(\beta)=\left[1 e^{-\mathrm{j} 2 \pi \frac{f(1)}{\lambda} \sin \beta} \cdots e^{-\mathrm{j} 2 \pi \frac{f(M-1)}{\lambda} \sin \beta}\right]^{\mathrm{T}}$. By performing DFT on $\widetilde{\mathbf{e}}(\beta)$, we obtain $\widetilde{\mathbf{c}}=\mathbf{F} \widetilde{\mathbf{e}}(\beta)$. Similar to (17), we have

$$
\left|\widetilde{c}_{n_{\max }}\right|=\left|\sum_{m=0}^{M-1} e^{-\mathrm{j} 2 \pi \widetilde{P}\left(n_{\max }, f(m)\right)}\right|,
$$

where

$$
\widetilde{P}\left(n_{\max }, f(m)\right)=\frac{f(m)}{\lambda} \sin \beta+\frac{m n_{\max }}{M} .
$$

Observe from (39) and (40) that unlike the linear elementpositioning, the index of the peak value in angular domain, $n_{\max }$, also depends on the nonlinear element-positioning function $f(m)$. Therefore, the E-DAR should be expressed as $\Delta\left(\beta_{1}, \beta_{2}, f(m)\right)$. However, the value of $\Delta\left(\beta_{1}, \beta_{2}, f(m)\right)$ is difficult to calculate. In order to facilitate a tractable and approximate calculation, we propose the concept of equivalent virtual angle-of-arrival in elevation domain (EV-E-AOA).
For the E-AOA $\beta$, we define the EV-E-AOA $\varphi$ as the following least squares (LS) solution

$$
\varphi=\arg \min _{\phi}\|\sin \phi \mathbf{m}-\sin \beta \mathbf{f}\|_{2},
$$

where $\mathbf{m}=\left[\begin{array}{lll}0 & 1 & \cdots\end{array}-1\right]^{\mathrm{T}}$ and $\mathbf{f}=[f(0) f(1) \cdots f(M-$ $1)]^{\mathrm{T}}$. The physical interpretation of EV-E-AOA is that $\varphi$ is the nearest E-AOA to approximate the real E-AOA $\beta$ for the given nonlinear element-positioning function and we have

$$
\varphi=\arcsin \left(\frac{\mathbf{m}^{\mathrm{T}} \mathbf{f}}{\mathbf{m}^{\mathrm{T}} \mathbf{m}} \sin \beta\right) .
$$

From the BS's point of view, by utilizing the nonlinear element-positioning function, the E-AOA $\beta$ is changed to another equivalent angle $\varphi$. Thus the E-DAR may be approximated as $\Delta\left(\beta_{1}, \beta_{2}, f(m)\right) \approx \Delta\left(\varphi_{1}, \varphi_{2}\right)$. This opens up a new avenue for enhancing the E-DAR by appropriately choosing $f(m)$, namely, by appropriately designing the structured NURA. Consequently, the achievable performance of the FDMIMO system is enhanced.

2) Exponent-based element-positioning function: A simple yet powerful nonlinear element-positioning function is the following power function:

$$
f_{\alpha}(m)=D \frac{m^{\alpha}}{\left(M_{y}-1\right)^{\alpha-1}}, 0 \leq m \leq M_{y}-1 .
$$

This nonlinear element-positioning function is specified by a single parameter, namely, the exponent $\alpha>0$, which determines the array structure of NURA. Fig. 3 illustrates the antenna-element distributions in the elevation-domain associated with $0<\alpha<1, \alpha=1$ and $\alpha>1$, given $M_{y}=4$. Clearly, for $\alpha=1$, the NURA degenerates to the URA, and $\alpha$ can be appropriately chosen to optimize the performance of the FD-MIMO system.

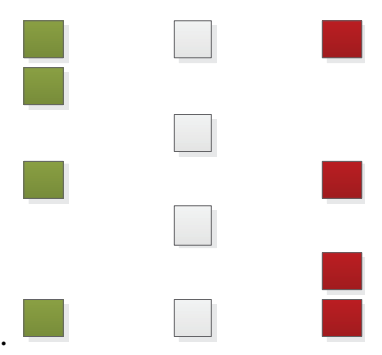

Fig. 3. Antenna-element distributions of the structured NURA in elevation domain given $M_{y}=4: f_{0<\alpha<1}(m)$ (left), $f_{\alpha=1}(m)$ (center), and $f_{\alpha>1}(m)$ (right).

Consider the senario discussed in Section II-B with $\beta_{\min }=0.173$ and $\beta_{\max }=0.611$. For the URA, we have $\Delta\left(\beta_{\min }, \beta_{\max }\right)=0.0298$. For the NURA having the exponentbased element-positioning function in elevation domain with $\alpha=1.2$, we have $\Delta\left(\varphi_{\min }, \varphi_{\max }\right)=0.0311$.

3) Exponential element-positioning function: Apart from the exponent-based positioning function of (43), there exists many nonlinear functions capable of creating different NURA devices. For example, another nonlinear element-positioning function is the following exponential function associated with the parameter $\mu>0$

$$
f_{\mu}(m)=D m \mu^{\left(\frac{m}{M_{y}-1}-1\right)}, 0 \leq m \leq M_{y}-1 .
$$


In particular, with $\mu=1$, this becomes the URA.

4) Tangent-based element-positioning function: Another nonlinear element-positioning function associated with the parameter $\nu \geq 0$ is defined by

$$
f_{\nu}(m)=D m \tan \left(\frac{\pi}{4} \frac{m^{\nu}}{\left(M_{y}-1\right)^{\nu}}\right), 0 \leq m \leq M_{y}-1
$$

This tangent-based element-positioning function subsumes the URA as the special case associated with $\nu=0$.

\section{B. Dynamic or Static Configuration}

Below we will mainly use the exponent-based elementpositioning function to illustrate the design of the structured NURA for optimizing the performance of the FD-MIMO. Our design procedure can be readily applied to other nonlinear element-positioning functions, such as the exponential and tangent-based functions.

1) Dynamic configuration: Given the set of users' A-AOAs and E-AOAs $\left\{\theta_{k}^{p}, \beta_{k}^{p}\right\}$ as well as the set of large-scale fading coefficients $\left\{\rho_{k}^{p}\right\}$, which specify a particular channel state $s$, then similar to (27), the instantaneous or channel state specific spectral efficiency of user $k$ is calculated as follows

$$
\begin{aligned}
\widetilde{C}_{k}(\alpha ; s) & =\log _{2}\left(1+\widetilde{\operatorname{SIR}}_{k}(\alpha ; s)\right) \\
& =\log _{2}\left(1+\frac{\left|\widetilde{\mathbf{h}}_{k}^{\mathrm{T}}(\alpha ; s) \widetilde{\mathbf{w}}_{k}(\alpha ; s)\right|^{2}}{\sum_{k^{\prime} \neq k}\left|\widetilde{\mathbf{h}}_{k}^{\mathrm{T}}(\alpha ; s) \widetilde{\mathbf{w}}_{k^{\prime}}(\alpha ; s)\right|^{2}}\right),
\end{aligned}
$$

where $\widetilde{\mathbf{h}}_{k}(\alpha ; s)$ denotes the column vectorization of $\widetilde{\mathbf{H}}_{k}(\alpha ; s)$ of (38) and $\widetilde{\mathbf{w}}_{k}(\alpha ; s)$ is the corresponding precoding vector. Here we have explicitly indicated that the channel matrix is a function of the specific elevation antenna-element distribution adopted, i.e., the value of $\alpha$, and of the channel state $s$. The instantaneous or channel-state-dependent spectral efficiency averaged over all the $K$ users is then given by

$$
\widetilde{C}(\alpha ; s)=\frac{1}{K} \sum_{k=1}^{K} \widetilde{C}_{k}(\alpha ; s) .
$$

The optimally structured NURA for the given exponent-based element-positioning function can thus be obtained by solving the following single-parameter optimization problem

$$
\alpha_{\mathrm{d}-\text { opt }}(s)=\arg \max _{\alpha>0} \widetilde{C}(\alpha ; s) .
$$

Clearly, both $\alpha_{\mathrm{d}-\mathrm{opt}}(s)$ and the achievable optimal spectral efficiency $\widetilde{C}\left(\alpha_{\mathrm{d} \text {-opt }}(s) ; s\right)$ vary according to the channel state $s$. Consequently, the optimal element-positioning function $f_{\alpha_{\mathrm{d}-\mathrm{opt}}(s)}(m)$ varies with the channel state $s$. Thus the NURA configuration has to be adjusted every time the channel state changes. This dynamic configuration of NURA (DC-NURA) requires a novel manufacturing process for the antenna arrays at the BS, which has not yet been defined in current wireless communication standards. This DC-NURA however provides the achievable performance upper bound for the NURA based FD-MIMO. Moreover, long and intensive research has produced various reconfigurable antennas [20], which are capable of adjusting the antenna structure during operational stage. In particular, the so-called liquid metal antennas [21]-[23] offer a promising solution of the dynamically reconfigurable antenna design for implementing our DC-NURA. Therefore, if the channel's coherence time is sufficiently long, it is practical to implement this DC-NURA. We believe in the near future, wireless standards will have provision for such reconfigurable antennas.

2) Static configuration: The current wireless standards do not have provision yet for periodically changing the antenna array's structure at the BS. In order to conform with a fixed or static array configuration, we can obtain the optimal static configuration of NURA (SC-NURA) by solving the following single-variable optimization problem

$$
\alpha_{\mathrm{s}-\mathrm{opt}}=\arg \max _{\alpha>0} E\{\widetilde{C}(\alpha ; s)\},
$$

where the ensemble expectation is over the distribution of the channel state $s$ or $\left\{\theta_{k}^{p}, \beta_{k}^{p} ; \rho_{k}^{p}\right\}$.

Because it is challenging to obtain the expression of $E\{\widetilde{C}(\alpha ; s)\}$, we opt for using a temporal average to approximate it. Specifically, we assume that a sufficiently large number of the channel state realizations or temporal snapshots, denoted by $\mathcal{S}=\left\{\left\{\theta_{k}^{p}(s), \beta_{k}^{p}(s) ; \rho_{k}^{p}(s)\right\}: 1 \leq s \leq S\right\}$, is available. Then we have

$$
\begin{aligned}
\widehat{C}(\alpha) & =\frac{1}{S} \sum_{s=1}^{S} \widetilde{C}(\alpha ; s)=\frac{1}{S K} \sum_{s=1}^{S} \sum_{k=1}^{K} \widetilde{C}_{k}(\alpha ; s) \\
& \approx E\{\widetilde{C}(\alpha ; s)\} .
\end{aligned}
$$

The optimal SC-NURA design for the given exponent-based element-positioning function is approximately solved by solving the following single-parameter optimization

$$
\widehat{\alpha}_{\mathrm{s}-\mathrm{opt}}=\arg \max _{\alpha>0} \widehat{C}(\alpha) .
$$

Clearly, for sufficiently large $S$, the solution of (51) is almost indistinguishable from the exact optimal solution $\alpha_{\mathrm{s}-\mathrm{opt}}$, and we have

$$
\lim _{S \rightarrow \infty} \widehat{\alpha}_{\mathrm{s}-\mathrm{opt}}=\alpha_{\mathrm{s}-\mathrm{opt}}
$$

Remarks: The set of channel state realizations $\mathcal{S}=$ $\left\{\left\{\theta_{k}^{p}(s), \beta_{k}^{p}(s) ; \rho_{k}^{p}(s)\right\}: 1 \leq s \leq S\right\}$ is not constituted by the actual channel state estimates. Rather, they are generated independently according to the underlying channel model distribution described in Section II, which is known. This is consistent with the true objective function $E\{\widetilde{C}(\alpha ; s)\}$ in which the expectation is with respect to the known underlying channel model distribution. Therefore, the SC-NURA design is entirely practical.

\section{Optimization}

The optimization problem (48) or (51) is a single-parameter optimization, which can be solved by many efficient algorithms. For the DC-NURA design, the objective function of (48) is likely to have multiple extremities owing to its instantaneous channel-state-dependent nature, while the objective function (50) of the SC-NURA design is likely to have a single extremity, because it is averaged over various channel states. These properties of the DC-NURA and SC-NURA design metrics will be investigated in the next section. Considering the 
TABLE I

PARAMETERS OF THE SIMULATED SINGLE-CELL MULTI-USER OUTDOOR SYSTEM.

\begin{tabular}{l|l}
\hline \hline Height of BS $l_{\mathrm{BS}}$ & $35 \mathrm{~m}$ \\
\hline Height of users $l$ & $1.5 \mathrm{~m}$ \\
\hline Number of users $K$ & $6,8,10$ \\
\hline Cell radius $r_{\max }$ & $250 \mathrm{~m}$ \\
\hline Minimum radius $r_{\min }$ & $50 \mathrm{~m}$ \\
\hline Path-loss exponent $\gamma$ & 3.5 \\
\hline Variance of shadow fading $\sigma_{z}^{2}$ & $8 \mathrm{~dB}$ \\
\hline URA array element spacing $D$ & $\lambda / 2$ \\
\hline Antenna array size $M \times M$ & $16 \times 16,32 \times 32,64 \times 64$ \\
\hline Number of paths $P$ & 20 \\
\hline Angle spread of A-AOAs & 180 degrees \\
\hline Angle spread of E-AOAs & 5 degrees \\
\hline \hline
\end{tabular}

potentially 'multi-peak' nature of the instantaneous spectral efficiency (47), we opt for using the search method of [24] to solve the optimization problems (48) and (51).

\section{PERformance Evaluation}

Our investigation mainly focuses on an outdoor user senario, and the parameters of this simulated outdoor system are listed in Table I. However, we will also simulate an indoor scenario with users in high rise buildings [25], [26]. The channels are generated according to (7). The number of channel states used in the simulation study is $S=1000$. Specifically, the communication environments, including the users' distribution, the large-scale fading coefficients and multi-path components, are randomly and independently generated $S=1000$ times.

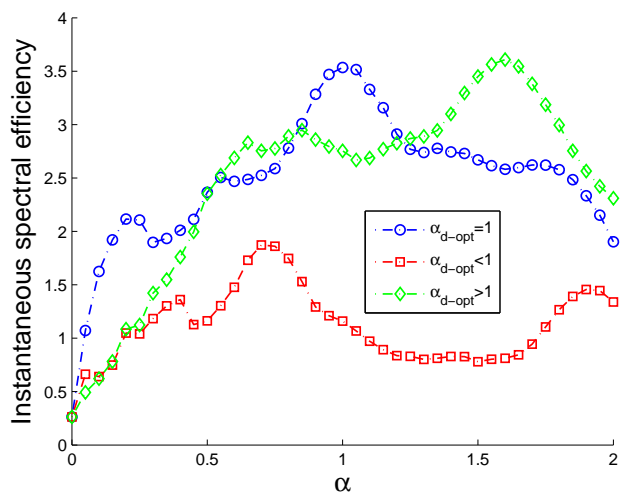

(a)

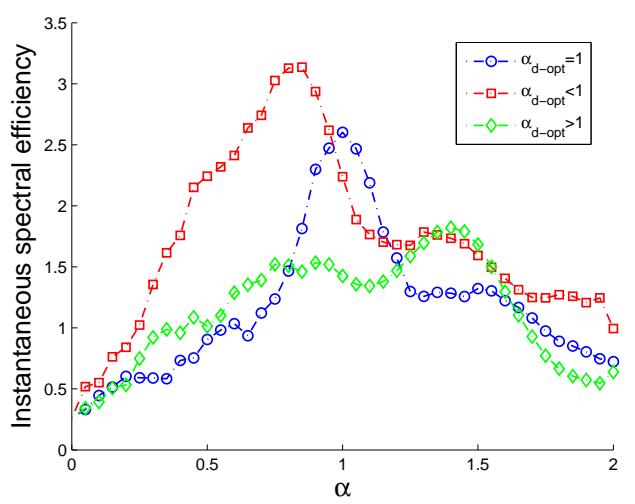

(b)

Fig. 4. Instantaneous spectral efficiency $\widetilde{C}(\alpha ; s)$ as a function of $\alpha$ for three channel states given the array size $64 \times 64$ and the number of users $K=6$ : (a) using the ZF-precoding, and (b) using the MF-precoding. Outdoor user scenario is simulated.

\section{A. Properties of NURA Design Metrics}

1) DC-NURA: We start with the exponent-based elementpositioning function (43). For a given channel state $s$, the spectral efficiency (47) is the function of $\alpha$. By solving the optimization (48) we obtain the optimal $\alpha_{\text {d-opt }}(s)$, which depends on $s$. For the array size of $M \times M=64 \times 64$ and $K=6$ users, Fig. 4 depicts the instantaneous spectral efficiency $\widetilde{C}(\alpha ; s)$ as a function of $\alpha$ for three different channel states. These three channel states are chosen to reflect all the three physical structures of Fig. 3. Observe that $\widetilde{C}(\alpha ; s)$ is generally of multi-peak nature and depending on the channel state, there exists an optimal NURA device whose spectral efficiency is significantly higher than that of the URA at $\alpha=1$. It can also be seen that for some of the channel states the URA is actually optimal.

Next, for the exponential element-positioning function (44), Fig. 5 illustrates $\widetilde{C}(\mu ; s)$ as the function of $\mu$ for three different channel states, while for the tangent-based element-positioning function (45), Fig. 6 shows $\widetilde{C}(\nu ; s)$ as the function of $\nu$ for three different channel states. It can be seen that both $\widetilde{C}(\mu ; s)$ and $\widetilde{C}(\nu ; s)$ exhibit multiple peaks and there exist optimal NURAs whose spectral efficiencies are significantly higher than those of the URA. Furthermore, for these two designs, the URA may actually be optimal for some specific channel states. For example, Fig. 5 indicates that there is an instantaneous spectral efficiency curve $\widetilde{C}(\mu ; s)$ which peaks at $\mu=1$, i.e., the URA is optimal for this particular channel state. Similarly,

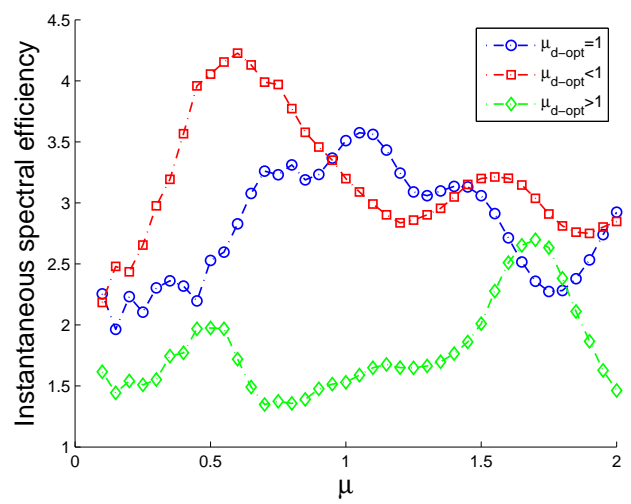

(a)

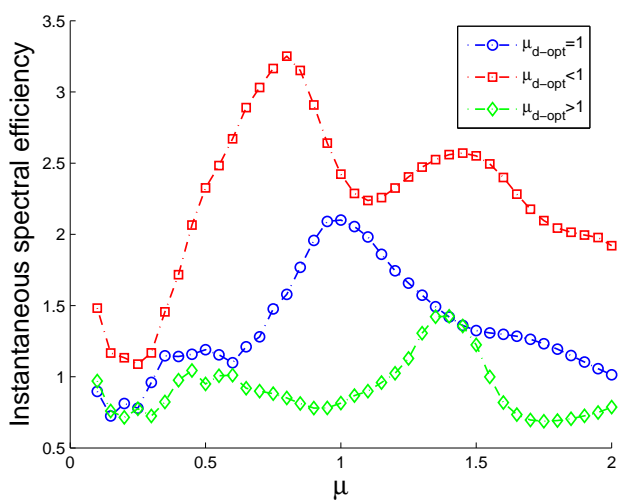

(b)

Fig. 5. Instantaneous spectral efficiency $\widetilde{C}(\mu ; s)$ as the function of $\mu$ for three channel states given the array size $64 \times 64$ and the number of users $K=6$ : (a) using the ZF-precoding, and (b) using the MF-precoding. Outdoor user scenario is simulated. 


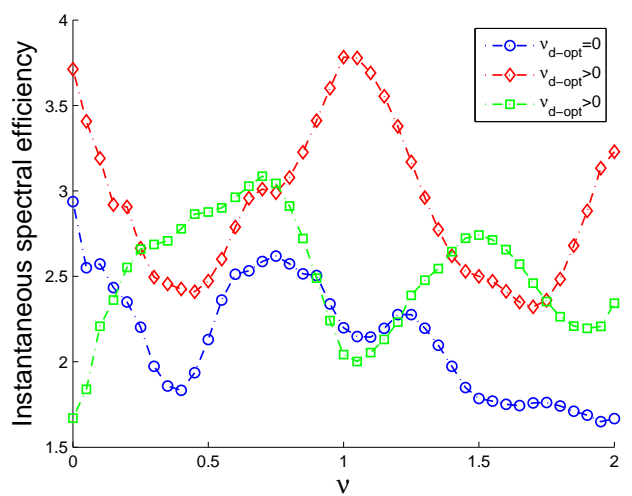

(a)

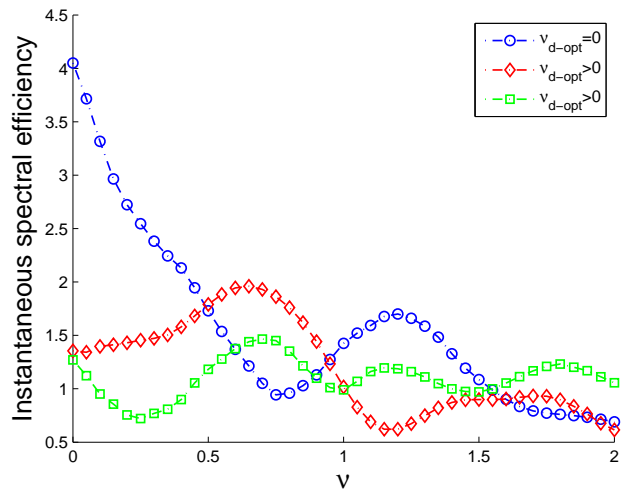

(b)

Fig. 6. Instantaneous spectral efficiency $\widetilde{C}(\nu ; s)$ as the function of $\nu$ for three channel states given the array size $64 \times 64$ and the number of users $K=6$ : (a) using the ZF-precoding, and (b) using the MF-precoding. Outdoor user scenario is simulated.

Fig. 6 shows that there is an instantaneous spectral efficiency curve $\widetilde{C}(\nu ; s)$ which exhibits a maximum value at $\nu=0$, hence the URA is optimal for this particular channel state.

It can be seen that for the DC-NURA, the designs based on all the three nonlinear element-positioning functions are capable of producing NURA devices that have a higher average spectral efficiency than the URA design.

2) SC-NURA: For the array size of $M \times M=64 \times 64$ and different numbers of users, Fig. 7 depicts the average spectral efficiency $\widehat{C}(\alpha)$ as the function of $\alpha$, while the average spectral efficiency $\widehat{C}(\mu)$ as the function of $\mu$ is shown in Fig. 8. It can be seen that for the SC-NURA, the average spectral efficiency generally has a single peak. Furthermore, for the exponentbased and exponential-based element-positioning functions, the optimal NURA designs significantly outperform the URA design. By contrast, the average spectral efficiency $\widehat{C}(\nu)$ is plotted as the function of $\nu$ in Fig. 9, demonstrating that $\widehat{C}(\nu)$ peaks at $\nu=0$, i.e. the URA is optimal.

The results of Figs. 7 and 8 demonstrate that for the SC-NURA, the optimal designs based on the exponent and exponential positioning functions are capable of producing NURAs that significantly outperform the URA design, but the results of Fig. 9 suggest that the tangent-based function is not particularly useful for designing SC-NURA. Moreover, observe from Figs. 7 and 8 that $\widehat{\alpha}_{\text {s-opt }}$ and $\widehat{\mu}_{\text {s-opt }}$ only change very slightly with different numbers of users. This near-stable property of the optimized SC-NURA is very attractive. In the

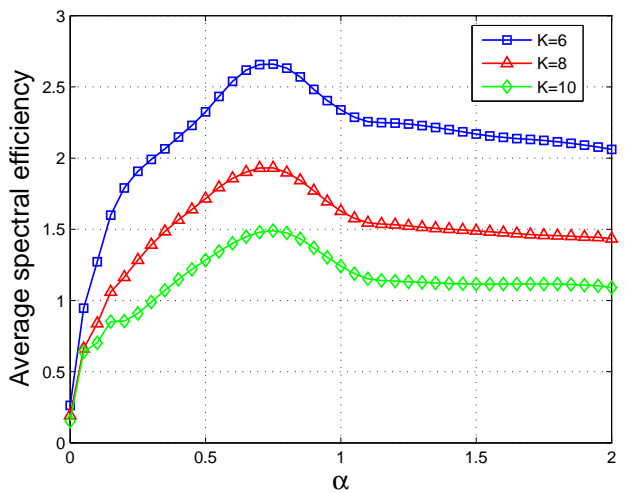

(a)

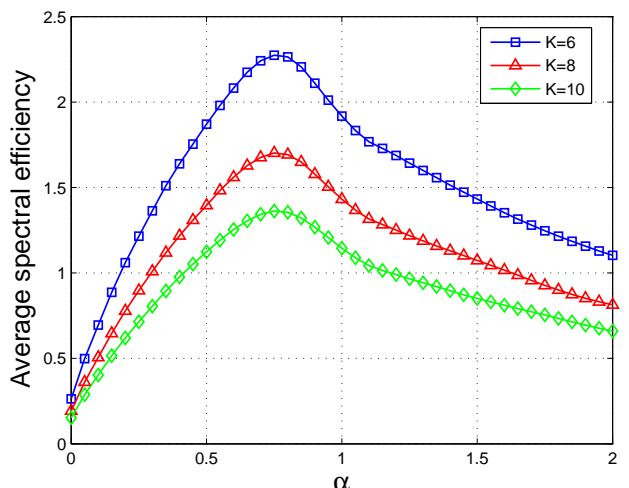

(b)

Fig. 7. Average spectral efficiency $\widehat{C}(\alpha)$ as the function of $\alpha$ for three different numbers of users $K$ and given the array size $64 \times 64$ : (a) using the ZF-precoding, and (b) using the MF-precoding. Outdoor user scenario is simulated.

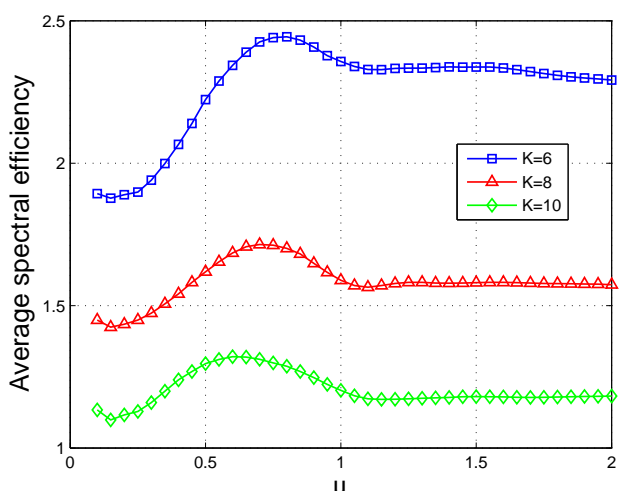

(a)

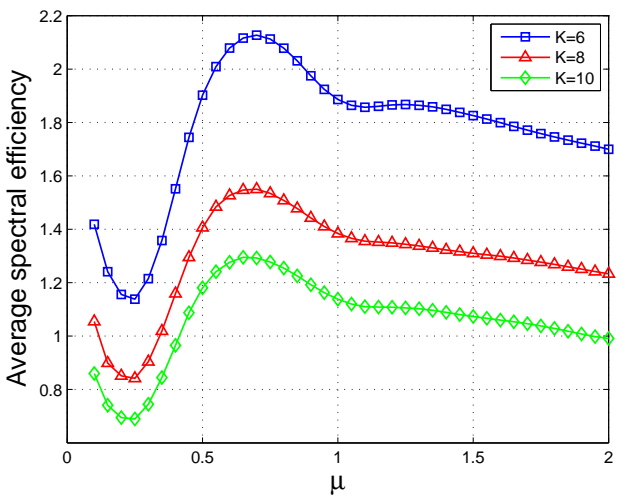

(b)

Fig. 8. Average spectral efficiency $\widehat{C}(\mu)$ as the function of $\mu$ for three different numbers of users $K$ and given the array size $64 \times 64$ : (a) using the ZF-precoding, and (b) using the MF-precoding. Outdoor user scenario is simulated. 


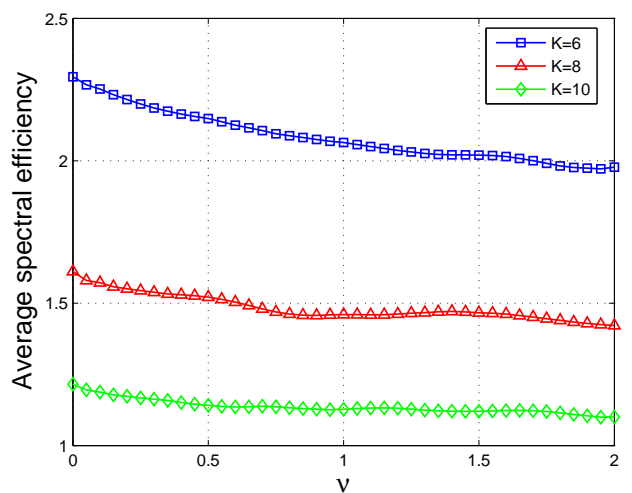

(a)

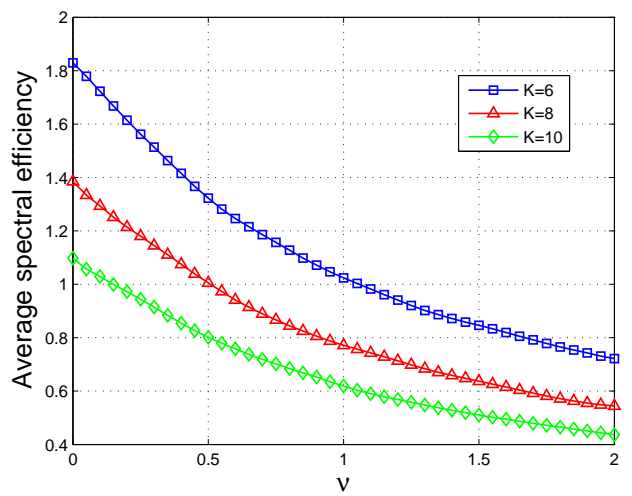

(b)

Fig. 9. Average spectral efficiency $\widehat{C}(\nu)$ as the function of $\nu$ for three different numbers of users $K$ and given the array size $64 \times 64$ : (a) using the ZF-precoding, and (b) using the MF-precoding. Outdoor user scenario is simulated.

network planning stage, the optimal SC-NURA configuration at the BS is designed with respect to a particular value of $K$. During the network operational life time, the number of users supported per resource block and per cell may change but we do not need to re-adjust the SC-NURA configuration.

\section{B. Spectral Efficiency Performance of NURA Designs}

For the array size of $M \times M=64 \times 64$ and for $K=6$ users, Fig. 10 compares the average spectral efficiency of both

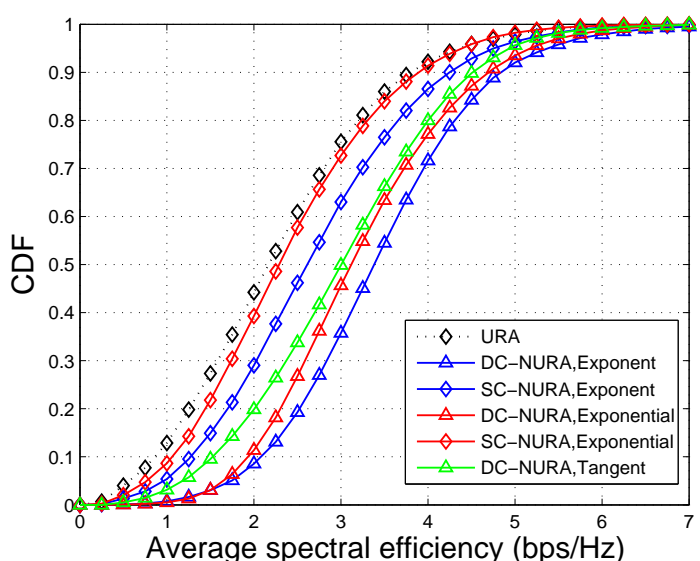

(a)

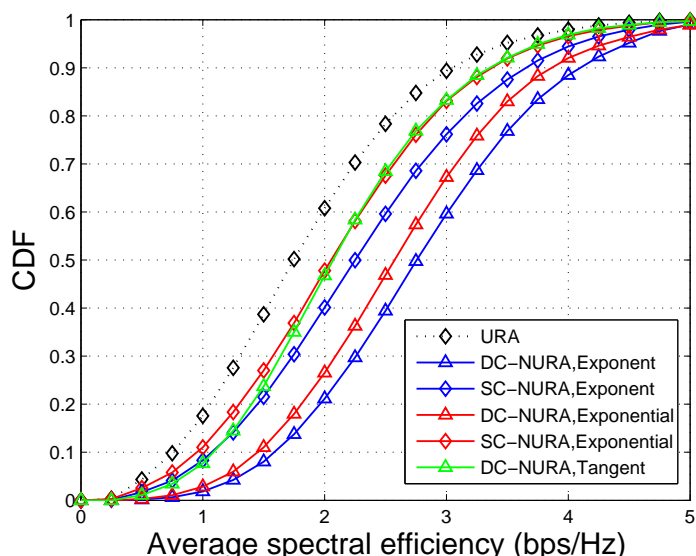

(b)

Fig. 10. CDFs of average spectral efficiencies for the URA design and various NURA designs given the array size $64 \times 64$ and the number of users $K=6$ : (a) using the ZF-precoding, and (b) using the MF-precoding. Outdoor user scenario is simulated.

the URA device and of various NURA devices by plotting the cumulative distribution functions (CDFs) of the average spectral efficiencies for these different array devices. It can be seen from Fig. 10 that both the DC-NURA and SCNURA designs outperform the standard URA design, with the DC-NURA attaining the best performance. For example, for

TABLE II

AVERAGE SPECTRAL EFFICIENCY (BPS/HZ) COMPARISON FOR DIFFERENT ARRAY DESIGNS IN OUTDOOR USER SCENARIO.

\begin{tabular}{|c|c|c|c|c|c|c|c|c|}
\hline \multirow{2}{*}{ Number of users } & \multirow{2}{*}{ Precoding } & \multirow{2}{*}{ Array size } & \multirow{2}{*}{ URA } & \multicolumn{2}{|c|}{ Exponent-based } & \multicolumn{2}{|c|}{ Exponential } & \multirow{2}{*}{$\begin{array}{c}\text { Tangent-based } \\
\text { DC-NURA }\end{array}$} \\
\hline & & & & DC-NURA & SC-NURA & DC-NURA & SC-NURA & \\
\hline \multirow{6}{*}{$K=6$} & \multirow{3}{*}{$\mathrm{ZF}$} & $16 \times 16$ & 1.71 & 2.28 & 1.87 & 2.44 & 1.82 & (1.95 \\
\hline & & $32 \times 32$ & 2.05 & 3.21 & 2.57 & 3.09 & 2.19 & 2.50 \\
\hline & & $64 \times 64$ & 2.27 & 3.44 & 2.72 & 3.26 & 2.40 & 3.02 \\
\hline & \multirow{3}{*}{ MF } & $16 \times 16$ & 0.96 & 1.16 & 1.01 & 1.18 & 1.02 & 1.10 \\
\hline & & $32 \times 32$ & 1.53 & 2.15 & 1.84 & 2.10 & 1.64 & 1.74 \\
\hline & & $64 \times 64$ & 1.84 & 2.81 & 2.32 & 2.69 & 2.10 & 2.16 \\
\hline \multirow{6}{*}{$K=8$} & \multirow{3}{*}{$\mathrm{ZF}$} & $16 \times 16$ & 1.54 & 2.05 & 1.60 & 2.06 & 1.57 & 1.82 \\
\hline & & $32 \times 32$ & 1.56 & 2.22 & 1.78 & 2.21 & 1.70 & 1.96 \\
\hline & & $64 \times 64$ & 1.60 & 2.51 & 1.86 & 2.35 & 1.73 & 2.16 \\
\hline & \multirow{3}{*}{ MF } & $16 \times 16$ & 0.73 & 0.85 & 0.76 & 0.84 & 0.76 & 0.79 \\
\hline & & $32 \times 32$ & 1.14 & 1.53 & 1.31 & 1.52 & 1.26 & 1.26 \\
\hline & & $64 \times 64$ & 1.38 & 2.08 & 1.73 & 1.95 & 1.64 & 1.62 \\
\hline \multirow{6}{*}{$K=10$} & \multirow{3}{*}{$\mathrm{ZF}$} & $16 \times 16$ & 1.28 & 1.84 & 1.32 & 1.83 & 1.34 & 1.66 \\
\hline & & $32 \times 32$ & 1.42 & 1.91 & 1.50 & 1.86 & 1.47 & 1.72 \\
\hline & & $64 \times 64$ & 1.43 & 1.93 & 1.53 & 1.93 & 1.51 & 1.87 \\
\hline & \multirow{3}{*}{ MF } & $16 \times 16$ & 0.58 & 0.65 & 0.60 & 0.64 & 0.59 & 0.63 \\
\hline & & $32 \times 32$ & 0.93 & 1.19 & 1.02 & 1.15 & 1.00 & 1.00 \\
\hline & & $64 \times 64$ & 1.11 & 1.66 & 1.38 & 1.59 & 1.29 & 1.28 \\
\hline
\end{tabular}


the ZF precoding and the exponent-based element-positioning function, only $38 \%$ of the average spectral efficiency is under $3 \mathrm{bps} / \mathrm{Hz}$ for the DC-NURA device, and about $45 \%$ of the average spectral efficiency is under $3 \mathrm{bps} / \mathrm{Hz}$ for the $\mathrm{SC}$ NURA device, while this proportion is increased to around $75 \%$ for the standard URA device. Similarly, for the classic MF precoding and the exponent-based element-positioning function, only $40 \%$ of the average spectral efficiency is under $2.5 \mathrm{bps} / \mathrm{Hz}$ for the DC-NURA design, and only $48 \%$ is under $2.5 \mathrm{bps} / \mathrm{Hz}$ for the SC-NURA design. By contrast, this is increased to almost $80 \%$ for the conventional URA design.

The average spectral efficiencies achieved by the different array designs are compared in Table II for various array sizes and numbers of users as well as for the two different precoding methods. As expected, the DC-NURA design attains the best performance on average, but this new NURA device design requires new type of reconfigurable antenna technology, as explained in the previous section. Our proposed SC-NURA device design is entirely practical and it significantly outperforms the standard URA device design in terms of its spectral efficiency, as clearly seen from Table II. In particular, the exponent-based element-positioning function is a suitable nonlinear function for designing the SC-NURA device. Table III shows the spectral efficiency gain of the SC-NURA design relying on the exponent-based function over the standard URA design.

We also investigate the influence of the channel noise $n_{k}$ on the achievable spectral efficiency. We define the signal to noise ratio (SNR) for user $k$ as the ratio of the desired signal power to the noise power according to

$$
\mathrm{SNR}_{k}=\frac{\left|\overline{\mathbf{h}}_{k}^{\mathrm{T}} \mathbf{w}_{k}\right|^{2}}{\sigma_{n_{k}}^{2}}
$$

where $\overline{\mathbf{h}}_{k}^{\mathrm{T}}$ and $\mathbf{w}_{k}$ are given in (25), while $\sigma_{n_{k}}^{2}$ denotes the power of the channel noise $n_{k}$. The system's SNR is then defined as

$$
\mathrm{SNR}=\frac{1}{K} \sum_{k=1}^{K} \mathrm{SNR}_{k}
$$

TABLE III

SPECTRAL EFFICIENCY GAIN OF THE SC-NURA DESIGN RELYING ON THE EXPONENT-BASED ELEMENT-POSITIONING FUNCTION OVER THE

\begin{tabular}{|c|c|c|c|}
\hline Array size & Number of users & Precoding & Spectral efficiency gain \\
\hline \multirow{6}{*}{$16 \times 16$} & & $\overline{\overline{Z F}}$ & $9 \%$ \\
\hline & $K=6$ & MF & $5 \%$ \\
\hline & & $\mathrm{ZF}$ & $4 \%$ \\
\hline & $K=8$ & MF & $4 \%$ \\
\hline & & $\mathrm{ZF}$ & $3 \%$ \\
\hline & $K=10$ & MF & $3 \%$ \\
\hline \multirow{6}{*}{$32 \times 32$} & & $\overline{\mathrm{ZF}}$ & $25 \%$ \\
\hline & $K=6$ & MF & $20 \%$ \\
\hline & & $\mathrm{ZF}$ & $14 \%$ \\
\hline & $K=8$ & MF & $15 \%$ \\
\hline & & $\mathrm{ZF}$ & $6 \%$ \\
\hline & $K=10$ & MF & $10 \%$ \\
\hline \multirow{6}{*}{$64 \times 64$} & & $\overline{\mathrm{ZF}}$ & $20 \%$ \\
\hline & $K=6$ & MF & $26 \%$ \\
\hline & & $\mathrm{ZF}$ & $16 \%$ \\
\hline & $K=8$ & MF & $25 \%$ \\
\hline & & $\mathrm{ZF}$ & $7 \%$ \\
\hline & $K=10$ & MF & $24 \%$ \\
\hline
\end{tabular}
STANDARD URA DESIGN IN OUTDOOR USER SCENARIO.

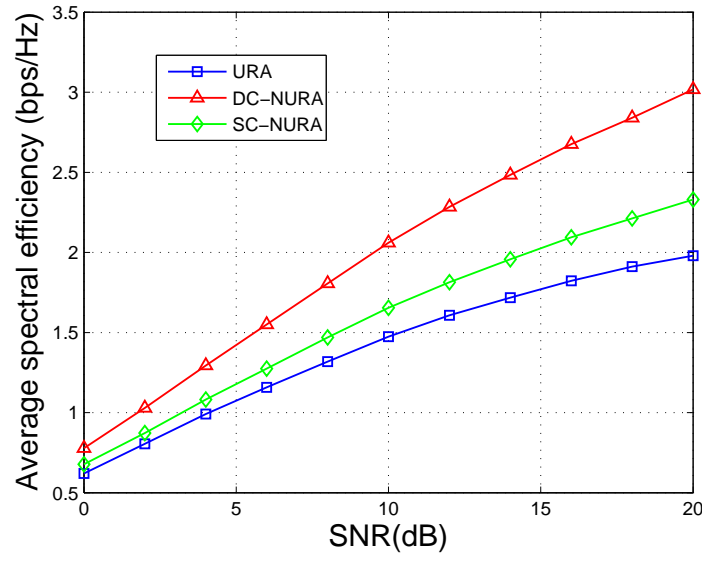

(a)

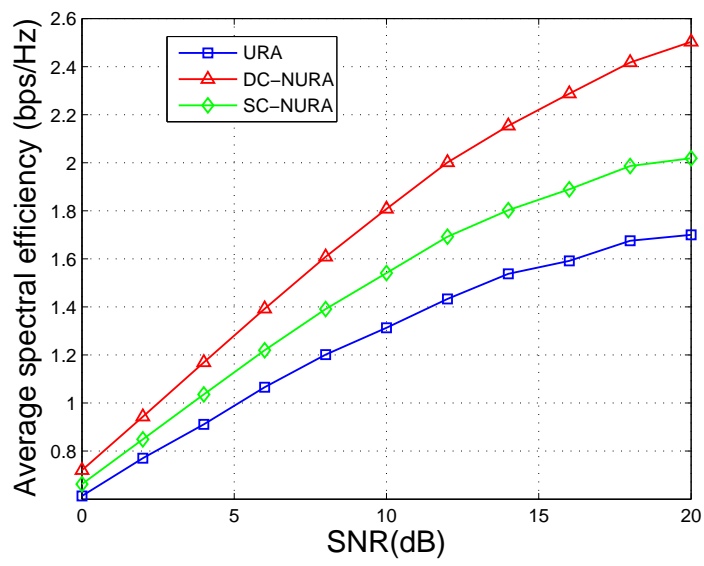

(b)

Fig. 11. Average spectral efficiencies versus the SNR for the URA as well as the SC-NURA and DC-NURA, both utilizing the exponent-based elementpositioning function, given the array size $64 \times 64$ and $K=6$ users: (a) using the ZF-precoding, and (b) using the MF-precoding. Outdoor user scenario is simulated.

In the simulation, we set every $\mathrm{SNR}_{k}=\mathrm{SNR}$ and assign the noise power $\sigma_{n_{k}}^{2}$ accordingly. Given the array size of $M \times M=$ $64 \times 64$ and $K=6$ users, Fig. 11 depicts the average spectral efficiency versus the SNR achieved by the URA as well as the SC-NURA and DC-NURA. Both NURA designs utilize the exponent-based element-positioning function.

\section{Indoor User Scenario}

All the above simulation results are obtained based on the outdoor user scenario. We now consider the case with indoor users in high rise scenario [25], [26], where the heights of users $l_{k}$ are generated according to

$$
l_{k}=3\left(n_{\mathrm{fl}}-1\right)+1.5 \mathrm{~m},
$$

in which $n_{\mathrm{fl}} \sim \mathcal{U}\left\{1,2, \cdots, N_{\mathrm{fl}}\right\}$ denotes the floor number and $N_{\mathrm{fl}} \sim \mathcal{U}\{4,5,6,7,8\}$ denotes the number of total floors, while $\mathcal{U}\{a, a+1, \cdots, b\}$ represents the integer random variable taking value from $\{a, a+1, \cdots, b\}$ with equal probability. The other parameters of the simulated indoor system are as given in Table I. Approximately, $80 \%$ of the users are indoor users, while the other $20 \%$ are outdoor users. For this indoor user scenario, Fig. 12 illustrates the average spectral efficiency versus the SNR achieved by the URA as well as 


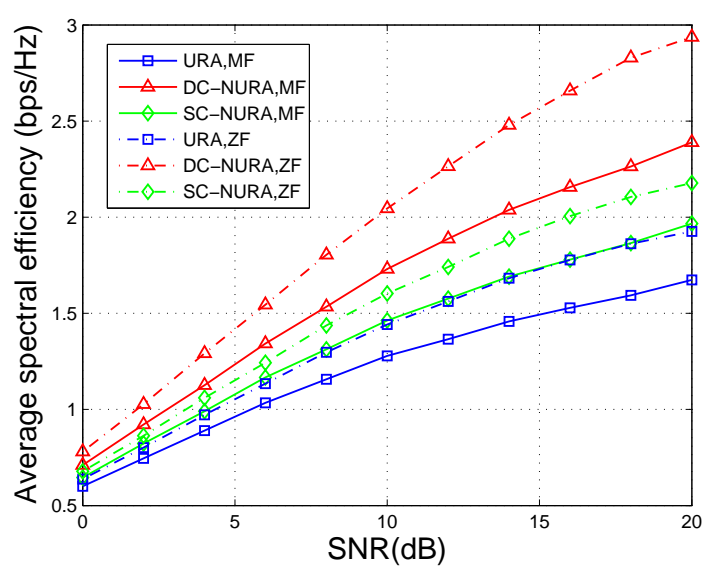

Fig. 12. Average spectral efficiencies versus the SNR for the URA as well as the SC-NURA and DC-NURA, both utilizing the exponent-based elementpositioning function, given the array size $64 \times 64$ and $K=6$ users. Indoor user scenario is simulated.

the SC-NURA and DC-NURA, given $M \times M=64 \times 64$ and $K=6$. Again, both the SC-NURA and DC-NURA adopt the exponent-based element-positioning function. Comparing Fig. 12 with Fig. 11, it can be seen that the average spectral efficiency is slightly lower than the outdoor-user case. This is not surprising because the E-AOAs may be overlapped in the indoor-user case which may cause additional inter-user interference. As expected, the proposed DC-NURA and SCNURA also outperform the URA in the indoor user scenario. Moreover, the spectral efficiency gain achieved by the NURA over the URA in the indoor-user case is very similar to that in the outdoor-user case, as can be seen from Table IV.

TABLE IV

SPECTRAL EFFICIENCY GAIN [BPS/Hz] OF THE NURA DESIGN RELYING ON THE EXPONENT-BASED ELEMENT-POSITIONING FUNCTION OVER THE URA DESIGN AT SNR $=20 \mathrm{DB}$, GIVEN $M \times M=64 \times 64$ AND $K=6$.

\begin{tabular}{c|cc|cc}
\hline & \multicolumn{2}{|c|}{ DC-NURA } & \multicolumn{2}{c}{ SC-NURA } \\
\hline & Indoor & Outdoor & Indoor & Outdoor \\
\hline $\mathrm{ZF}$ & 1.0 & 1.0 & 0.3 & 0.3 \\
$\mathrm{MF}$ & 0.7 & 0.8 & 0.3 & 0.3 \\
\hline
\end{tabular}

\section{Further discussions}

All the above analyses and simulation results for the standard URA and our proposed NURA ignore the mutual coupling between antenna elements, because for the URA the 'clean' elevation- and azimuth-domain steering vectors of (5) and (6) are adopted, while for the NURA the 'clean' elevationand azimuth-domain steering vectors of (37) and (6) are used. Clearly, the achievable capacity calculated using the clean steering vectors will be slightly different from the real capacity of the real system, where the antenna elements are mutually coupled.

It is well known that the mutual coupling is negligible only when the antenna elements are well separated from one another, and the effects of the mutual coupling become significant for the element spacing smaller than $2.5 \lambda$ in the URA scenario. Generally, the smaller the element spacing, the stronger the mutual coupling. It is difficult to say whether the NURA design has stronger or weaker mutual coupling than the URA of half wavelength spacing. This is because unlike the URA, the mutual couping of the NURA is nonuniform. More specifically, the overall spatial size of the NURA in the elevation direction is the same as that of the URA. As a result of the nonlinear element spacing, the top-part (or bottom-part) of the NURA has larger antenna spacing than half-the-wavelength, while the other part has smaller antenna spacing than half-the-wavelength, Therefore, about half of the NURA have weaker mutual coupling, while the other half have stronger mutual coupling than the URA of half wavelength spacing.

A meaningful question to ask here is whether the mutual coupling is a bad thing or a good thing. The answer is of course dependent on our design objective. For a diversity scheme, for example, the mutual coupling is detrimental, as it dramatically reduces the achievable diversity gain. In order to attain the full diversity gain, we have to place the antenna elements far apart, with at least $10 \lambda$ apart, so that there is no coupling and the signals at different antenna elements are mutually independent. For beamforming, which is the topic of this paper, the signals at different antenna elements have to be correlated for it to work. Explicitly, for beamforming we need the mutual couplings between the antenna elements. This is why for beamforming applications, the array element spacing is typically chosen to be $D=0.5 \lambda$ for one-dimensional ULA as well as for two-dimensional URA.

For one-dimensional ULA, experimental results have shown that the mutual coupling in closely spaced ULAs is actually beneficial to the achievable capacity or spectral efficiency [27]-[30]. Specifically, the results of [27] suggest that when the mutual coupling is taken into account, the capacity become similar for the element spacings of $D=0.5 \lambda$ and $D=0.1 \lambda$. The work [28] finds that for the spacings between $0.1 \lambda$ and $0.3 \lambda$, mutual coupling provides a clear capacity benefit, while for the spacings below $0.1 \lambda$, mutual coupling degrades the capacity, compared to the array spacing of $0.5 \lambda$. The studies [29], [30] conclude that the capacity for spacings between $0.2 \lambda \leq D<0.5 \lambda$ is larger than that of $D \geq 0.5 \lambda$. For two-dimensional antenna arrays, the results of this paper have convincingly demonstrated that our NURA achieves a significant higher capacity than the standard URA having the same size, albeit the capacity expressions for both the URA and NURA are based on clean steering vectors.

We further point out that for one-dimensional ULA, there exist some techniques [31]-[34] which can model the effects of mutual coupling. Further research is warranted to investigate how to extend these one-dimensional array modeling methods to two-dimensional antenna arrays, including the standard URA and our novel NURA.

\section{Conclusions}

A novel structured non-uniformly spaced rectangular antenna array design has been proposed for FD-MIMO systems. Our new contribution has been twofold. Firstly, we have derived a generic discrete angular measure that quantitatively determines the antenna array's resolution in both the azimuth and elevation domains. Based on this DAR measure, we have shown that the resolution of the URA for E-AOAs can be an 
order of magnitude lower than that for A-AOAs, which reveals the root cause of the URA's significant performance loss in comparison to a ULA of the same size. This has naturally motivated us to propose alternative antenna arrays relying on nonuniform element-positioning devices in the elevation domain. In order to make the problem sufficiently simple and tractable, we have opted for a structured NURA design, which optimizes the nonuniform distribution of elevation-domain antenna elements by a single control parameter. Our DC-NURA design offers the achievable performance upper bound for the NURA based FD-MIMO, but it requires new reconfigurable antenna technologies since this NURA structure has to change every time the channel state changes. By contrast, our SCNURA design is entirely practical based on the current fixed antenna structure design. Extensive simulation results have demonstrated that our optimally designed structured NURA based on the proposed nonlinear antenna-element-positioning function significantly outperforms the standard URA in terms of its achievable spectral efficiency.

\section{REFERENCES}

[1] E. G. Larsson, O. Edfors, F. Tufvesson, and T. L. Marzetta, "Massive MIMO for next generation wireless systems," IEEE Commun. Mag., vol. 52, no. 2, pp. 186-195, Feb. 2014.

[2] H. Q. Ngo, E. G. Larsson, and T. L. Marzetta, "Energy and spectral efficiency of very large multiuser MIMO systems," IEEE Trans. Commun., vol. 61, no. 4, pp. 1436-1449, Apr. 2013.

[3] T. L. Marzetta, "Noncooperative cellular wireless with unlimited numbers of base station antennas," IEEE Trans. Wirel. Commun., vol. 9, no. 11 , pp. 3590-3600, Nov. 2010.

[4] F. Rusek, D. Persson, B. K. Lau, E. G. Larsson, T. L. Marzetta, O. Edfors, and F. Tufvesson, "Scaling up MIMO: Opportunities and challenges with very large arrays," IEEE Signal Proc. Mag., vol. 30, no. 1, pp. 40-60, Jan. 2013.

[5] Y.-H. Nam, B. L. Ng, K. Sayana, Y. Li, J. Zhang, Y. Kim, and J. Lee, "Full-dimension MIMO (FD-MIMO) for next generation celluar technology," IEEE Commun. Mag., vol. 51, no. 6, pp. 172-179, Jun. 2013.

[6] X. Cheng, B. Yu, L. Yang, J. Zhang, G. Liu, Y. Wu and L. Wan, "Communicating in the real world: 3D MIMO," IEEE Wirel. Commun., vol. 21, no. 4, pp. 136-144, Aug. 2014.

[7] Y. Kim, H. Ji, J. Lee, Y.-H. Nam, B. L. Ng, I. Tzanidis, Y. Li and J. Zhang, "Full dimension MIMO (FD-MIMO): The next evolution of MIMO in LTE systems," IEEE Wirel. Mag., vol. 21, no. 3, pp. 92-100, Jun.2014.

[8] A. Kammoun, H. Khanfir, Z. Altman, M. Debbah, and M. Kammoun, "Preliminary results on 3D channel modeling: From theory to standardization," IEEE J. Sel. Areas Commun., vol. 32, no. 6, pp. 1219-1229, Jun. 2014.

[9] Q.-U.-A. Nadeem, A. Kammoun, M. Debbah, and M. S. Alouini, "3D massive MIMO systems: Modeling and performance analysis," IEEE Trans. Wirel. Commun., vol. 14, no. 12, pp. 6926-6939, Dec. 2015.

[10] N. Seifi, J. Zhang, R. W. Heath, T. Svensson and M. Coldrey, "Coordinated 3D beamforming for interference management in cellular networks," IEEE Trans. Wirel. Commun., vol. 13, no. 10, pp. 53965410, Oct. 2014.

[11] R1-150560, "Codebook for 2D antenna arrays," 3GPP TSG RAN WG1\#80, Feb. 2015.

[12] A. Adhikary, J. Nam, J.-Y. Ahn, and G. Caire, "Joint spatial division and multiplexing - the large-scale array regime," IEEE Trans. Inf. Theory, vol. 59, no. 10, pp. 6441-6463, Oct. 2013.

[13] Z. Wang, W. Liu, C. Qian, S. Chen, and L. Hanzo, "Two-dimensional precoding scheme for 3D massive MIMO," IEEE Trans. Vehicular Technology, DOI: 10.1109/TVT.2016.2622009, pp. 1-7, 2016.

[14] D. Ying, F. W. Vook, T. A. Thomas, D. J. Love and A. Ghosh, "Kronecker product correlation model and limited feedback codebook design in a 3D channel model," in Proc. ICC 2014 (Sydney, NSW), Jun. $10-14,2014$, pp. $5865-5870$.

[15] R1-150381, "Discussions on FD-MIMO codebook enhancements," 3GPP TSG RAN WG1\#80, Feb. 2015.
[16] R1-150516, "Performance of Kronecker-based CSI feedback for EBF/FD-MIMO," 3GPP TSG RAN WG1\#80.

[17] Z. Wang, P. Zhao, C. Qian, and S. Chen, "Location-aware channel estimation enhanced TDD based massive MIMO," IEEE Access, vol. 4 pp. 7828-7840, 2016

[18] H. Dahrouj and W. Yu, "Coordinated beamforming for the multicell multi-antenna wireless system,", IEEE Trans. Wirel. Commun., vol. 9, no. 5, pp. 1748-1759, May 2010.

[19] L. You, X. Gao, X.-G. Xia, N. Ma and Y. Peng, "Pilot reuse for massive MIMO transmission over spatially correlated rayleigh fading channels," IEEE Trans. Wirel. Commun., vol. 14, no. 6, pp. 3352-3366, Jun. 2015.

[20] J. Costantine, Y. Tawk, S. E. Barbin, and C. G. Christodoulou, "Reconfigurable antennas: design and applications," Proc. IEEE, vol. 103, no. 3, pp. 424-437, Mar. 2015.

[21] S. Cheng, A. Rydberg, K. Hjort, and Z. Wu, "Liquid metal stretchable unbalanced loop antenna," Appl. Phys. Lett., vol. 94, no. 14, pp. 1441031-144103-3, 2009.

[22] M. Wang, C. Trlica, M. R. Khan, M. D. Dickey, and J. J. Adams, "A reconfigurable liquid metal antenna driven by electrochemically controlled capillarity," J. Appl. Phys., vol. 117, no. 19, pp. 1949011-194901-5, 2015.

[23] K.-F. Tong, C. B. Fortuny, and J. Bai, "Low cost 3D-printed monopole fluid antenna," in Proc. ISAP 2015 (Hobart, Tasmania, Australia), Nov. 9-12, 2015, pp. $1-4$.

[24] I. Moser, "Hooke-Jeeves revisited," in Proc. CEC 2009 (Trondheim, Norway), May 18-21, 2009, pp. 2670-2676.

[25] 3GPP TR 36.873, "Study on 3D channel model for LTE (Release 12)."

[26] 3GPP TR 36.897, "Study on Elevation Beamforming/Full-Dimension (FD) MIMO for LTE (Release 13)."

[27] T. Svantesson and A. Ranheim, "Mutual coupling effects on the capacity of multielement antenna systems," in Proc. ICASSP 2001 (Salt Lake City, USA), May 7-11, 2001, pp. 2485-2488.

[28] J. W. Wallace and M. A. Jensen, "The capacity of MIMO wireless systems with mutual coupling," in Proc. VTC 2002-Fall (Vancouver, Canada), Sep. 24-28, 2002, pp. 696-700

[29] V. Jungnickel, V. Pohl, and C. von Helmolt, "Capacity of MIMO systems with closely spaced antennas," IEEE Commun. Lett., vol. 7, no. 8, pp. 361-363, Aug. 2003.

[30] X. Liu and M. E. Bialkovwski, "Effect of antenna mutual coupling on MIMO channel estimation and capacity," Int. J. Antennas Propagat., Vol. 2010, pp. 1-9, 2010.

[31] C. Waldschmidt, J. V. Hagen, and W. Wiesbeck, "Influence and modelling of mutual coupling in MIMO and diversity systems," in Proc. 2002 Int. Symp. Antennas Propagat. Society (San Antonio, USA), Jun. 16-21, 2002, pp. 190-193.

[32] C. Volmer, J. Weber, R. Stephan, K. Blau, and M. A. Hein, "An eigen-analysis of compact antenna arrays and its application to port decoupling," IEEE Trans. Antennas Propagat., vol. 56, no. 2, pp. 360370, Feb. 2008

[33] R. Janaswamy, "Effect of element mutual coupling on the capacity of fixed length linear arrays," IEEE Antennas Wireless Propagat. Lett., vol. 1, pp. 157-160, 2002 .

[34] J. W. Wallace and M. A. Jensen, "Mutual coupling in MIMO wireless systems: A rigorous network theory analysis," IEEE Trans. Wireless Commun., vol. 3, no. 4, pp. 1317-1325, Jul. 2004.

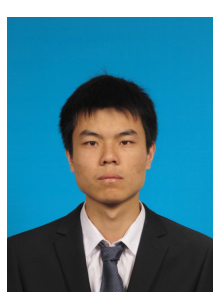

Wendong Liu received the B.S. degree (Hons.) from Tsinghua University, Beijing, China, in 2015. $\mathrm{He}$ is currently pursuing the Ph.D. degree with the Department of Electronic Engineering, Tsinghua University. His research interests include massive MIMO, full-dimension MIMO and millimeter-wave communications. 


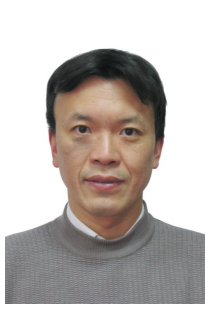

Zhaocheng Wang (M'09-SM'11) received the B.S., M.S., and Ph.D. degrees from Tsinghua University, Beijing, China, in 1991, 1993, and 1996, respectively.

From 1996 to 1997, he was a Postdoctoral Fellow with Nanyang Technological University, Singapore. From 1997 to 1999, he was with OKI Techno Centre (Singapore) Pte. Ltd., Singapore, where he was first a Research Engineer and later became a Senior Engineer. From 1999 to 2009, he was with Sony Deutschland $\mathrm{GmbH}$, where he was first a Senior Engineer and later became a Principal Engineer. He is currently a Professor of Electronic Engineering with Tsinghua University and serves as the Director of Broadband Communication Key Laboratory, Tsinghua National Laboratory for Information Science and Technology (TNlist). He has authored or coauthored over 120 journal papers. $\mathrm{He}$ is the holder of 34 granted U.S./EU patents. He coauthored two books, one of which, Millimeter Wave Communication Systems, was selected by IEEE Series on Digital and Mobile Communication (Wiley-IEEE Press). His research interests include wireless communications, visible light communications, millimeterwave communications, and digital broadcasting. He is a Fellow of the Institution of Engineering and Technology. $\mathrm{He}$ served as the Associate Editor of IEEE Transactions on Wireless Communications (2011-2015) and the Associate Editor of IEEE Communications Letters (2013-2016), and has also served as Technical Program Committee Co-Chair of various international conferences.

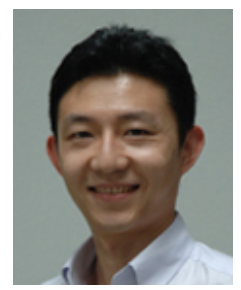

Chen Sun (S'02-M'05-SM'12) obtained the PhD degree in electrical engineering from Nanyang Technological University, Singapore, in 2005.

From August 2004 to May 2008, he was a researcher at ATR Wave Engineering Laboratories, Japan, working on adaptive beamforming and direction finding algorithms of parasitic array antennas as well as theoretical analysis of cooperative wireless networks. In June 2008, he joined the National Institute of Information and Communications Technology (NICT), Japan, as an expert researcher working on distributed sensing and dynamic spectrum access in TV white space. Since then he has been contributing to IEEE 1900.6 standard, IEEE 802.11a standard and Wi-Fi Alliance specifications for Wi-Fi networks in TV white space. In 2012 he joined Sony China Research Laboratory where he is the head of Wireless Network Research Department. He served as the technical editor of the IEEE 1900.6 standard and received the IEEE Standard Association Award for his contribution in 2011. He also received the IEEE 802.11af Outstanding Contributions Award in 2012 and the IEEE-SA Standards Board acknowledges with appreciation to the development of IEEE1900.6a standard in March 2014. He served as the rapporteur of ETSI EN EN 303143 from 2013 to 2015 . Currently he serves as the technical editor of the IEEE 802.19.1a task group.

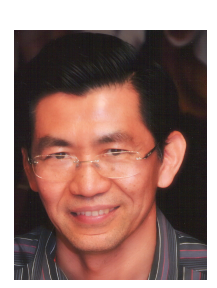

Sheng Chen (M'90-SM'97-F'08) received his BEng degree from the East China Petroleum Institute, Dongying, China, in 1982, and his $\mathrm{PhD}$ degree from the City University, London, in 1986, both in control engineering. In 2005, he was awarded the higher doctoral degree, Doctor of Sciences (DSc), from the University of Southampton, Southampton, UK.

From 1986 to 1999, He held research and academic appointments at the Universities of Sheffield, Edinburgh and Portsmouth, all in UK. Since 1999, he has been with School of Electronics and Computer Science, the University of Southampton, UK, where he currently holds the post of Professor in Intelligent Systems and Signal Processing. Dr Chen's research interests include adaptive signal processing, wireless communications, modelling and identification of nonlinear systems, neural network and machine learning, intelligent control system design, evolutionary computation methods and optimisation. He has published over 550 research papers. Dr. Chen is a Fellow of the United Kingdom Royal Academy of Engineering, a Fellow of IET, a Distinguished Adjunct Professor at King Abdulaziz University, Jeddah, Saudi Arabia, and an ISI highly cited researcher in engineering (March 2004).

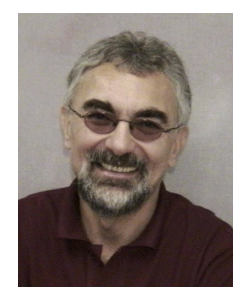

Lajos Hanzo (F'04) received the D.Sc. degree in electronics in 1976, the Ph.D. degree in 1983, and the Doctor Honoris Causa degree from the Technical University of Budapest in 2009. He also received the Ph.D. degree (Hons.) from the University of Edinburgh in 2015.

During his 40 -year career in telecommunications, he has held various research and academic positions in Hungary, Germany, and the U.K. From 2008 to 2012, he was the Editor-in-Chief of the IEEE Press and a Chaired Professor with Tsinghua University, Beijing. Since 1986, he has been with the School of Electronics and Computer Science, University of Southampton, U.K., as the Chair in Telecommunications. He has successfully supervised $110 \mathrm{Ph}$.D. students, co-authored 20 John Wiley/IEEE Press books in mobile radio communications totalling in excess of 10000 pages, authored over 1500 research entries at the IEEE Xplore. He has over 25000 citations. He is directing a 100 Strong Academic Research Team, working on a range of research projects in the field of wireless multimedia communications sponsored by the industry, the Engineering and Physical Sciences Research Council, U.K., the European Research Councils Advanced Fellow Grant, and the Royal Society's Wolfson Research Merit Award. He is an enthusiastic supporter of industrial and academic liaison and offers a range of industrial courses. He is also a fellow of the Royal Academy of Engineering, the Institution of Engineering and Technology, and the European Association for Signal Processing. He is a Governor of the IEEE VTS. He acted as the TPC Chair and General Chair of the IEEE conferences, presented keynote lectures, and received a number of distinctions. 